\title{
The FASEB Journal
}

Copy of e-mail Notification

Your article (\# 08-113464) from The FASEB Journal is available for downloading.

$====$

The FASEB Journal is published by the Federation of American Societies for Experimental Biology (FASEB)

Dear author,

Please click on this URL address http://rapidproof.cadmus.com/RapidProof/retrieval/index.jsp then Login: your e-mail address

Password: ---- (You may copy and paste this case-sensitive password.)

The site contains one (1) file. You will need to have Adobe Acrobat(r) Reader software to read it. This software is freely available for download at www.adobe.com.

Please print the PDF file of your proof and read it carefully. Indicate changes or corrections in the margin of the page. If you need to provide the editorial office with a revised figure, please draw and " $\mathrm{X}$ " through the incorrect figure and write "NEW FIGURE FILE REQUIRED" in the margin. The compositor will contact the corresponding author to obtain the file(s).

Within 48 hours after receiving this message, please fax forms to the following fax numbers:

Fax PROOF CORRECTIONS to: 1-717-738-9479

Proof corrections sent by any other means or faxed to any other number may result in substantial publication delays.

Fax PUBLICATION FORMS to: 1-301-634-7809

Publication forms sent by any other means or faxed to any other number may result in substantial publication delays.

NOTE: Proofs or publication forms retained by the author for an excessive length of time may not be published online in a timely way and may need to be scheduled for a later print issue.

If you have any problems or questions, please contact me. Always include your article number in all correspondence.

Sincerely,

Mary Kiorpes Hayden

FASEB Office of Publications

9650 Rockville Pike

Bethesda, MD 20814-3998

phone: 301-634-7151

fax: 301-634-7809

mhayden@faseb.org 


\section{The FASEB Journal}

The Journal of the Federation of American Societies for Experimental Biology

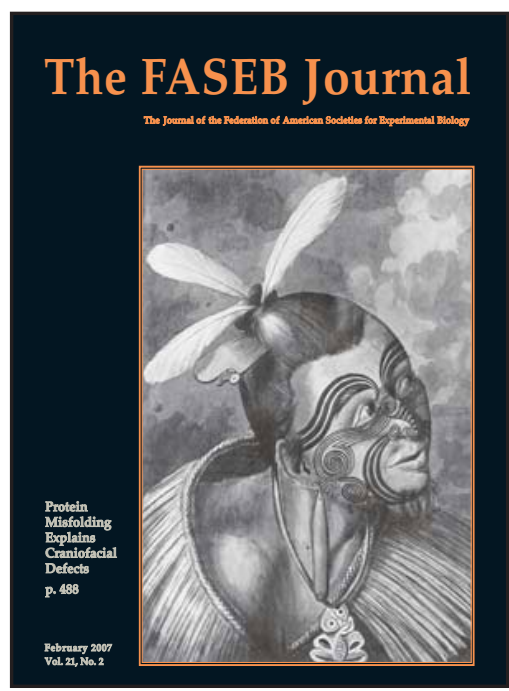

As an engaged-and engaging-source for insights into new science, The FASEB Journal publishes the best of peer-reviewed work in experimental biology from around the world.

As disciplines in the life sciences continue to overlap, readers are drawn to The FASEB Journal for its interdisciplinary coverage and perspective. The FASEB Journal has become a preferred venue for the latest research reports and reviews of epigenetics, iRNA mechanics, histone acetylation, nitric oxide signaling, eicosanoid biochemistry, angiogenesis, tumor suppressor genes, apoptosis, cytoskeletal function, human stem cell research, and more!

The FASEB Journal is a journal of all the Life Sciences and the Life of Science.

Join us at www.fasebj.org.

\begin{tabular}{|llll|}
\hline 2008 Subscription Prices & $\begin{array}{l}\text { United } \\
\text { States }\end{array}$ & $\begin{array}{l}\text { Canada/ } \\
\text { Mexico }\end{array}$ & $\begin{array}{l}\text { Rest } \\
\text { of World* }\end{array}$ \\
FASEB Member Print \& Online & $\$ 119$ & $\$ 146$ & $\$ 227$ \\
FASEB Member Online Only & $\$ 70$ & $\$ 70$ & $\$ 70$ \\
Individual Print \& Online & $\$ 205$ & $\$ 231$ & $\$ 313$ \\
Student Online Only & $\$ 45$ & $\$ 45$ & $\$ 45$ \\
Institution Print \& Online & $\$ 896$ & $\$ 923$ & $\$ 1005$ \\
Institution Online Only & $\$ 833$ & $\$ 833$ & $\$ 833$
\end{tabular}

Payment Options-Please circle desired subscription type in price chart above.

$\square$ VISA (13 or 16 Digits) $\square$ MasterCard (16 Digits) $\square$ EuroCard $\square$ American Express $\square$ Discover

Card Number Expiration Date

Cardholder Name Signature

Organization Telephone

Credit card orders may be faxed to (301) 634-7099 or emailed to staff@dues.faseb.org.

$\square$ Check or Money Order (US Currency Only - Drawn on US Bank)

Please send my subscription to:

Name

Organization

Address

City/State/ZIP-Postal Code

Telephone

Email

Mail to: The FASEB Journal, Dues \& Subscriptions, Room L-2310, 9650 Rockville Pike, Bethesda, MD 20814-3998 USA Call: 800-43-FASEB, Ext. 7029 (1-800-433-2732 x 7029 US only) or (301) 634-7029 Fax: (301) 634-7099 


\section{AUTHOR INSTRUCTIONS \\ Copyright Transfer and \\ Publication Costs Approval Form}

All authors are required to sign the following copyright transfer and cost agreement form prior to publication. If you have not yet submitted this form to the editorial office, please fax it to 301-6347809 as soon as possible. Multiple forms may be submitted for the same article. Instructions for calculating publication charges are also attached for your convenience. Please read that sheet carefully. Do not fax your estimate sheet to the editorial office. 
The Journal of the Federation of American Societies for Experimental Biology

\section{Mandatory Copyright Transfer and Publication Costs Approval Form}

Manuscript No.:

Title:

Author Names (Please Print All Names):

\section{Signatures Below Certify Compliance With the Following Statements:}

Copyright Transfer. In consideration of the acceptance of the above work for publication, I do herby assign and transfer to the Federation of American Societies for Experimental Biology (FASEB) all rights, titles, and interest in and to the copyright in The FASEB Journal. This includes preliminary display/posting of the abstract of the accepted article in electronic form before publication. The journal grants the author permission to provide a copy of the accepted manuscript to NIH upon acceptance for Journal publication, with public release in PubMed Central twelve months after final print publication by The FASEB Journal.

This form must be signed by all authors. If any changes in authorship (order, deletions, or additions) occur after the manuscript is submitted, agreement by all authors for such changes must be on file with FASEB. An author's name may only be removed at his/her own request and with written consent from all of the other authors, as well as final approval by the Editor-in-Chief. Material prepared by employees of the US Government in the course of their official duties cannot be copyrighted; work prepared by employees of the British or British Commonwealth government in the course of their official duties is subject to Crown Copyright and cannot be transferred to FASEB. Nevertheless, authors must sign the form to indicate acceptance of all terms other than copyright transfer.

O Please check if this article was written as part of the official duties of an employee of the US government.

O Please check if this article was written as part of the official duties of an employee of the British or British Commonwealth government.

\section{Authorship Responsibilities. I attest that:}

1. the manuscript is not currently under consideration, in press, or published elsewhere, and the research reported will not be submitted for publication elsewhere until a final decision has been made as to its acceptability by The FASEB Journal (posting of submitted material on a web site or by any other electronic means may be considered prior publication - note this in your cover letter);

2. the manuscript is truthful, original work without fabrication, fraud, or plagiarism;

3. I have made an important scientific contribution to the study and am thoroughly familiar with the primary data and;

4. I have read the complete manuscript and take responsibility for the content and completeness of the manuscript and understand that I share responsibility if the paper, or part of the paper, is found to be faulty or fraudulent.

Conflict of Interest Disclosure. All funding sources supporting the work and all institutional or corporate affiliations of mine are acknowledged. Except as disclosed on a separate attachment, I certify that I have no commercial associations (e.g., consultancies, stock ownership, equity interests, patent-licensing arrangements, etc.) that might pose a conflict of interest in connection with the submitted article, and that I accept full responsibility for the conduct of the trial, had full access to all the data, and controlled the decision to publish.

Author Fees. I agree to pay the following publication charges. Page charges are $\$ 80$ per printed page for the first 8 pages; $\$ 160$ per page for the 9th page and beyond. Color figures are $\$ 350$ each. Articles containing eight or more figures and/or tables will be charged an additional $\$ 150$ per figure and table. Supplemental files uploaded to the journal website are $\$ 100$ for each file. All figures are in U.S. dollars.

Author Signatures. For more than 10 authors, use an extra sheet. Multiple forms are acceptable.

1. Print Name:

2. Print Name:

3. Print Name:

4. Print Name:

5. Print Name:

6. Print Name:

7. Print Name:

8. Print Name:

9. Print Name:

10. Print Name:
Signature:

Signature:

Signature:

Signature:

Signature:

Signature:

Signature:

Signature:

Signature:

Signature:
Date:

Date:

Date:

Date:

Date:

Date:

Date:

Date:

Date:

Date:

Signed forms should be faxed to 301-634-7809 or scanned and emailed to Mary Hayden at mhayden@faseb.org. E-mail questions to Mary Hayden at mhayden@faseb.org or call 301-634-7151. 


\section{AUTHOR INSTRUCTIONS}

\section{Calculating Publication Costs}

The FASEB Journal now uses an online bill pay system for publication and reprint order fees. The corresponding author will receive a separate e-mail containing a link to a web page where charges can be paid by credit card. The e-mail link also will provide information about invoices, check payment, and wire transfers.

Publication charges are calculated based on the final version of the article and not this proof. Color figures have the word "COLOR" in the margins. Grayscale (black and white) figures have no tag. If you wish to change a color figure to grayscale, cross out the word "color" and write in "grayscale." Please note that figures are published the same way in the online and print versions of the journal. Authors may not publish figures in color online while publishing the same figures in grayscale in print or vice-versa. Authors wishing to reduce costs by combining supplemental files should contact Mary Hayden at mhayden@faseb.org.

For your convenience and records, the following table should help you estimate publication charges. Please account for any changes you have made to your proofs. Please do not fax this sheet to the editorial office. Your charges will be calculated automatically based on the final article.

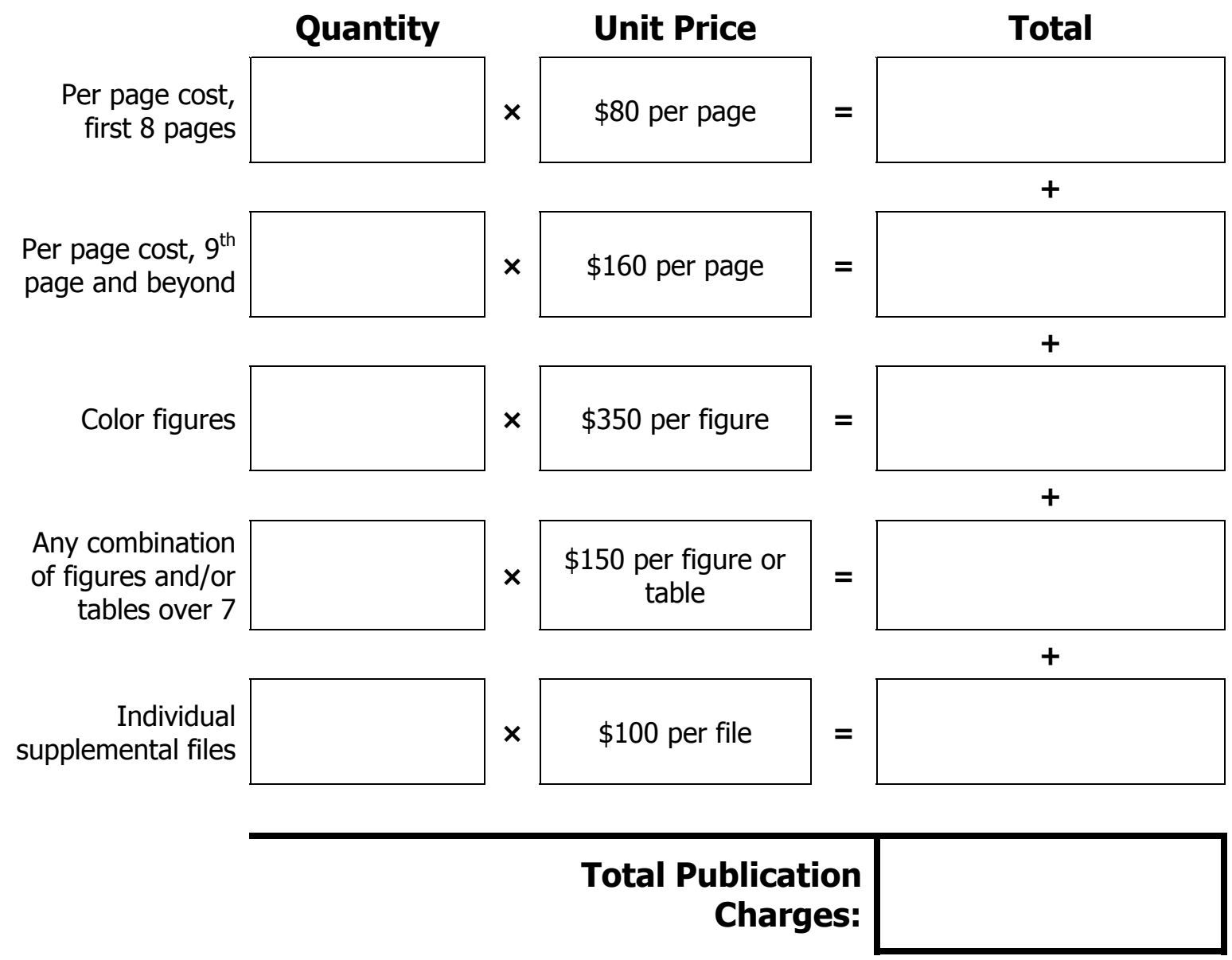

This sheet is for estimation purposes only. Final costs are calculated using the final printed version of the article. All prices are in U.S. dollars. Open access fees are calculated separately. Reprints can be purchased when paying publication charges online. 


\section{AUTHOR INSTRUCTIONS Page Proofs}

Please review following article proof and fax corrections to: 1-717-738-9479. Page proofs returned in any other way or faxed to any other number may lead to substantial publication delays. Please keep copies for your files.

Color figures have the word "COLOR" in the margins. Grayscale (black and white) figures have no tag. If you wish to change a color figure to grayscale, cross out the word "color" and write in "grayscale." (See below.) Publication charges will be calculated based on your changes to this proof. Please note that figures are published the same way in the online and print versions of the journal. Authors may not publish figures in color online while publishing the same figures in grayscale in print or vice-versa.
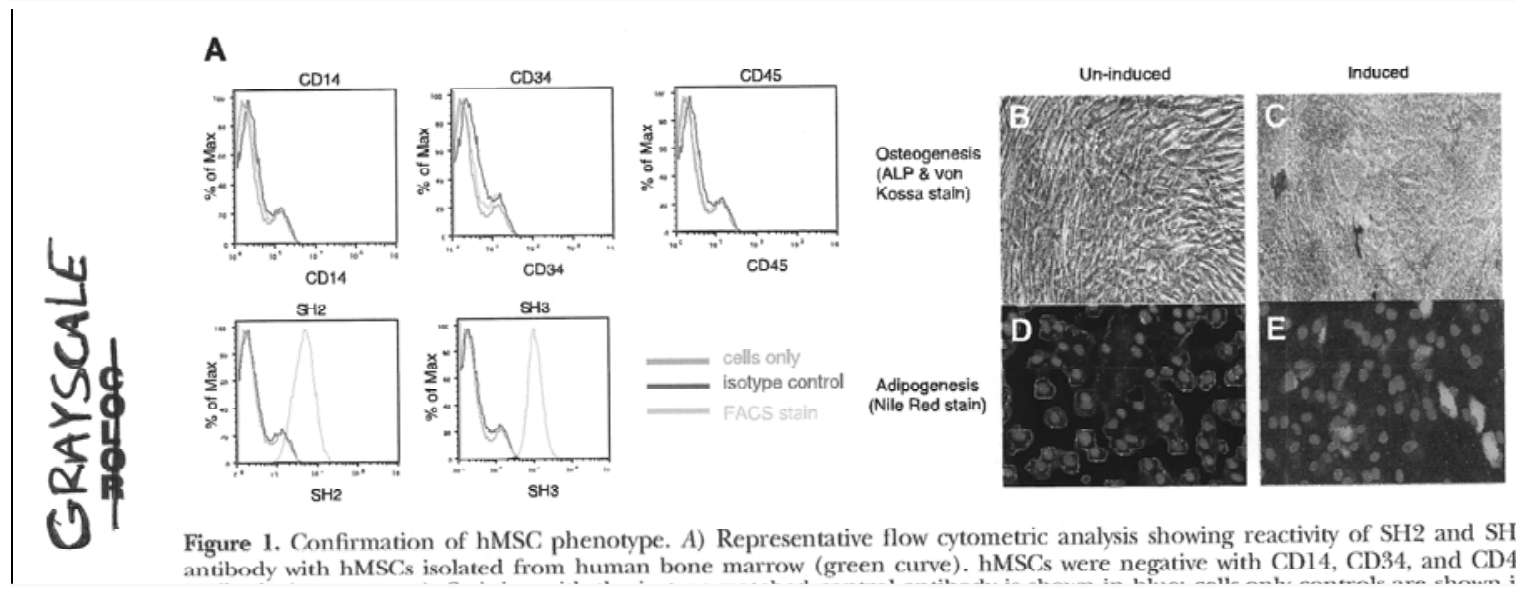

Figure 1. Confirmation of hMSC phenotype. A) Representative flow cytometric analysis showing reactivity of SH2 and SH3 antibody with hMSCs isolated from human bone marrow (green curve). hMSCs were negative with CD14, CD34, and CD45

NOTE: If you need to send new figure files to the editorial office, please draw an " $X$ " through the figure and write "NEW FIGURE FILE REQUIRED" in the margin (see below). The editorial office will contact the corresponding author to obtain the necessary file(s).

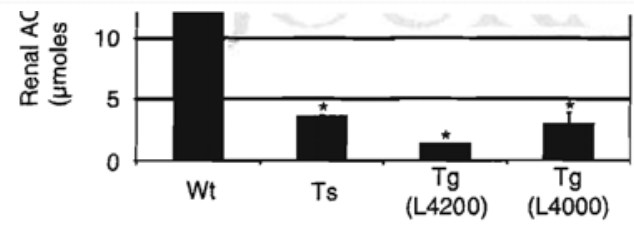

Figure 2. ACE activity assay. Age-matched, adult serum $(A)$, lung extract $(B)$, and kidney extract $(C)$ were prepared from Wt and strain Ts, $\mathrm{Tg}$ (Line 4200) and $\mathrm{Tg}$ (Line 4000) mice as described in the Experimental Procedures. Lung or kidney protein extract $(25 \mu \mathrm{g})$ or serum $(1 \mu \mathrm{l})$ was assayed for ACE activity, measured as $\mu$ moles of His-Leu generated by cleavage of Ang I analog Hip-His-Leu in $1 \mathrm{~h}$ at $37^{\circ} \mathrm{C}$. Each bar represents the average value from triplicate samples from five mice of the same genotype. Error bars indicate the $95 \%$ CI for each data sets. ${ }^{*} P \leq 0.001^{* *} P \leq 0.0005$, ${ }^{* * *} P \leq 0.00005$ vs. Wt control strain mice.

sion in the Wt mouse. In contrast, as expected, transgenic $\mathrm{SACE}$ or gACE was not expressed in the proximal tubules (Fig. 5).

To correlate the level of ACE activity observed in the lung samples obtained from the Ts and $\mathrm{Tg}$ strain mice (Fig. 2) with the level of ACE produced in the vascular endothelial cells of these same strains, we repeated the restored fertility (30). All of our other Ace-/- mice expressing SACE in sperm (Ps strain), or sACE in other somatic tissues (i.e., vasculature, kidneys or serum), remained sterile $(29,30)$. Similarly, male fertility was

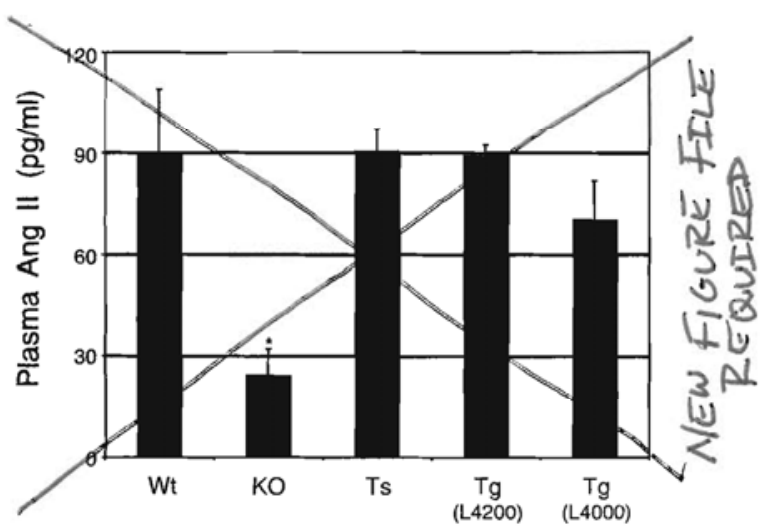

Figure 3. Plasma Ang II measurements. Plasma $(1 \mathrm{ml})$ pooled from 4 adult mice of the same age, sex, and genotype was assayed for Ang II levels as described in Experimental Procedures. Each data point is the average of duplicate measure- 


\section{AUTHOR INSTRUCTIONS}

\section{Proofreader's Marks}

Use a pen with black, blue, or red ink. Make two marks for every correction: one in text and one in the margin. A mark in text may be a caret $(\wedge)$ or a line drawn through the character or word to be deleted. If there are several changes to be made in one line, arrange them in sequence left to right separated by a slash line. For the same correction repeated in one line, write the correction and a slash for each repetition (example: $\mathrm{A} / /$ to add two commas. For number corrections, slash through the wrong digits in the text, write the correct digits in the $\begin{array}{llll}\text { margin, and circle the correct number in the margin: } 7 \$ \$ 0 & 52 & 7520 .\end{array}$

\begin{tabular}{|c|c|c|c|}
\hline Mark & Meaning & Example & Result \\
\hline II & Align & $\|<$ copyfitting & copyfitting \\
\hline 2 & Apostrophe & proofreaders marks & proofreader's marks \\
\hline man & Boldface & point & point \\
\hline$[/]$ & Brackets & $\Delta$ letterspacing $[/]$ & [letterspacing] \\
\hline$\equiv$ & Caps & cantion & CAPTION \\
\hline$c / 1 c$ & Caps and lowercase & $\sqrt{\mathrm{STIFICATION}} c / / c$ & Justification \\
\hline$][$ & Center & ]column [ & column \\
\hline$\infty$ & Change copy as indicated & eleven fonts use & 11 fonts \\
\hline 2 & Close up & head line & headline \\
\hline$\therefore /$ & Colon & subheag $_{h}: /$ & subhead: \\
\hline$\hat{\jmath}$ & Comma & indentation $/$ & indentation, \\
\hline$\mu$ & Delete & net hardly & hardly \\
\hline 2 & Delete and close up & keröing & kerning \\
\hline$\frac{1}{m}$ & Em dash & descende $\frac{1}{m}$ and & descender-and \\
\hline !) & Exclamation point & ascendeh I/ & ascender! \\
\hline$=1$ & Hyphen & demis bold & demi-bold \\
\hline$\square$ & Indent & D character & character \\
\hline$\wedge$ & Insert & typeface $A$ library & typeface library \\
\hline - & Italicize & leading & leading \\
\hline$/ / c$ & Lowercase & KXXDDARAPAH IC & typography \\
\hline E & Move left & E composition & composition \\
\hline ב & Move right & tex & text \\
\hline 9 & New paragraph & display: Type & $\begin{array}{c}\text { display. } \\
\text { Type }\end{array}$ \\
\hline$(/)$ & Parentheses & $\Delta$ roman & (roman) \\
\hline - & Period & backslante & backslant. \\
\hline (2) & Query & itelic (?SP) & italic \\
\hline$? /$ & Question mark & boldfacen ?/ & boldface? \\
\hline $4 / 2$ & Quotation marks & "condensed" & "condensed" \\
\hline stẹt & Retain original & compressede stet & compressed \\
\hline 2 & Run on & $\frac{\text { new faces }}{c \text { are }}$ & new faces are \\
\hline ;/ & Semicolon & expanded if & expanded; \\
\hline$=\mathrm{sc}$ & Small caps & PUNCTUATION & PUNCTUATION \\
\hline \# & Space & linblength & line length \\
\hline No & Space evenly & im the news & in the news \\
\hline Ntr & Transpose & runduspnd tr & runaround \\
\hline (x) & Type is bad & Orisual $\infty$ & visual \\
\hline$\subseteq \omega f$ & Wrong font & cursive wf & cursive \\
\hline
\end{tabular}




\title{
The FASEB Journal • Research Communication
}

\section{The histone subcode: poly(ADP-ribose) polymerase-1 (Parp-1) and Parp-2 control cell differentiation by regulating the transcriptional intermediary factor TIF1 $\beta$ and the heterochromatin protein HP1 $\alpha$}

AQ: 1

\author{
Delphine Quénet,* Véronique Gasser, ${ }^{*}$ Laetitia Fouillen, ${ }^{\ddagger}$ Florence Cammas, ${ }^{\dagger}$ \\ Sarah Sanglier-Cianferani, ${ }^{\ddagger}$ Régine Losson, ${ }^{\dagger}$ and Françoise Dantzer*,1 \\ *Département IDG, UMR7175, ESBS, Illkirch, France; ${ }^{\dagger}$ IGBMC, Illkirch, France; and ${ }^{\ddagger}$ Laboratoire de \\ Spectrométrie de Masse Bio-organique, UMR7178, ECPM, Strasbourg, France
}

\begin{abstract}
Recent advances reveal emerging unique functions of poly(ADP-ribose) polymerase-1 (Parp-1) and Parp-2 in heterochromatin integrity and cell differentiation. However, the chromatin-mediated molecular and cellular events involved remain elusive. Here we describe specific physical and functional interactions of Parp- 1 and Parp-2 with the transcriptional intermediary factor (TIF1及) and the heterochromatin proteins (HP1) that affect endodermal differentiation. We show that Parp-2 binds to TIF1 $\beta$ with high affinity both directly and through HP1 $\alpha$. Both partners colocalize at pericentric heterochromatin in primitive endoderm-like cells. Parp-2 also binds to HP1 $\beta$ but not to HP1 $\gamma$. In contrast Parp-1 binds weakly to TIF1 $\beta$ and HP1 $\beta$ only. Both Parps selectively poly(ADP-ribosyl)ate HP1 $\alpha$. Using shRNA approaches, we provide evidence for distinct participation of both Parps in endodermal differentiation. Whereas Parp-2 and its activity are required for the relocation of TIF1 $\beta$ to heterochromatic foci during primitive endodermal differentia-

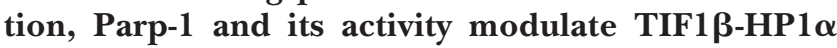
association with consequences on parietal endodermal differentiation. Both Parps control TIF1 $\beta$ transcriptional activity. In addition, this work identifies both Parps as new modulators of the HP1-mediated subcode histone.-Quénet, D., Gasser, V., Fouillen, L., Cammas, F., Sanglier-Cianferani, S., Losson, R., Dantzer, F. The histone subcode: poly(ADP-ribose) polymerase-1 (Parp-1) and Parp-2 control cell differentiation by regulating the transcriptional intermediary factor TIF1 $\beta$ and the heterochromatin protein HP1 $\alpha$. FASEB J. 22, 000-000 (2008)
\end{abstract}

AQ: 2 Key Words: epigenetics

DyNAMIC REORganization of heterochromatic compartments and the epigenetic changes that are associated are currently recognized as important regulators of gene silencing that accompanies diverse cellular processes, including cell differentiation (1). This finding is further determined by the compartmentalization of specific protein complexes containing histones and nonhistone proteins, chromatin-remodeling and chromatin modifying enzymes, and by the post-translational modifications of histones defined as the histone code $(2-4)$. However, how these activities are coregulated remains unknown.

A broad distribution of heterochromatin (HC) is observed at pericentromeric regions known to play an important role in gene silencing (4). Its epigenetic nature is defined by methylation of cytosine-phosphateguanine $(\mathrm{CpG})$, hypoacetylation of histones, and methylation at lysine 9 of histone H3 (meH3K9), which is necessary for the enrichment in heterochromatin protein 1 (HP1) (5). Three distinct mammalian HP1s have been characterized: HP1 $\alpha$ and HP1 $\beta$ are primarily found within centromeric $\mathrm{HC}$; whereas $\mathrm{HP} 1 \gamma$ is enriched at euchromatic sites (6). These proteins participate in chromatin packaging and have a well-established function in HC-mediated silencing. The structure of the HP1 proteins consists of an N-terminal chromodomain (CD) that binds meH3K9 and the histone fold motif of histone $\mathrm{H} 3$, a central hinge domain (hinge) that displays RNA/DNA binding activities, and a C-terminal chromoshadow domain (CSD) recognized as a proteinprotein interaction domain $(7,8)$. It has been shown recently that HP1s are also targets of post-translational modifications similar to those described for histones, which define the existence of an HP1-mediated histone subcode (9). Notably, HP1s interact with proteins involved in transcriptional regulation through a specific PxVxL motif called HP1 box, among them the transcription intermediary factor $($ TIF1 $\beta)(10-12)$. TIF1 $\beta$ functions as a corepressor for the large family of Krüppel-associated box (KRAB)- domain-containing zinc-finger proteins and acts as a molecular scaffold to coordinate various activities that regulate chromatin structure and dynamics (13-15). In addition, TIF1 $\beta$

\footnotetext{
${ }^{1}$ Correspondence: Département IDG, UMR7175, ESBS, 67412 Illkirch, France. E-mail: fdantzer@esbs.u-strasbg.fr doi: $10.1096 /$ fj.08-113464
} 
exerts essential functions in early embryonic development (16) and spermatogenesis (17).

The mouse embryonal carcinoma F9 cells represent a well-established model of endodermal differentiation that can be induced to differentiate into primitive-endoderm-like (PrE) cells when grown as a monolayer in the presence of retinoic acid (RA) and subsequently in parietal endoderm-like (PE) cells when grown in the presence of both RA and dibutyryl cAMP (18). In this system, TIF1 $\beta$-HP1s association plays an essential role in the relocation of TIF $1 \beta$ from euchromatin to $\mathrm{HC}$ and in the progression through differentiation by regulating the expression of endoderm-specific genes (19).

Another regulatory mechanism that controls chromatin structure and integrity is the modification of histones and other nuclear proteins by poly(ADP-ribose) polymers catalyzed by poly(ADP-ribose) polymerases (Parps). Among the 17 members of the Parp family, Parp-1 and Parp-2 heterodimerize, share common binding partners and have been described as active players in the single-strand break/base excision repair process (20). Parp-1- and Parp-2-deficient mice and cells are very sensitive to both ionizing radiation and alkylating agents, thus supporting a role of both Parps in the cellular response to DNA damage (21). Moreover, Parp- $1^{-/}$Parp- $2^{-/}$embryos die at gastrulation, demonstrating the crucial role of poly(ADP-ribosyl)ation during embryonic development (21). Several lines of evidence support the view that Parp-1 and Parp-2 play prominent roles in the maintenance of constitutive and facultative $\mathrm{HC}$ integrity, with, however, the emergence of specific functions for Parp-2. Both proteins localize to telomeres (22), centromeres (23, 24), and rDNA (25), where they interact and regulate specific partners. Parp- $2^{-/-}$cells exhibit DNA damageinduced kinetochore defects; whereas the Parp- $1^{+/-}$ Parp- $2^{-/-}$background displays specific female embryonic lethality associated with $\mathrm{X}$ chromosome instability (21).

Interestingly, both Parp-1 and Parp-2 were also suggested to play critical roles in the progression through differentiation. In the developing mammalian central nervous system, Parp-1 serves roles in transcriptional events required for neuronal differentiation (26). We have recently described the appearance of specific spontaneous defects in differentiation processes, including adipogenesis (27), spermiogenesis (28), and T-lymphocyte maturation (29) in the Parp- $2^{-/-}$mice. However, the chromatin-mediated molecular mechanisms by which Parp-1 and Parp-2 may control differentiation have not yet been elucidated.

In this work we provide the first evidence for physical and functional selective interactions between Parp-2, Parp-1, TIF1 $\beta$, and HP1 $\alpha$ that have fundamental implications in $\mathrm{HC}$ structure and/or function governing endodermal differentiation. We show that Parp-2 physically binds to TIF $1 \beta$ with high affinity both directly and through HP1 $\alpha$. Both proteins relocate to pericentric HC throughout differentiation. We also identified a direct interaction of Parp-2 with HP1 $\beta$. A weaker but significant direct interaction of Parp-1 with TIF1 $\beta$ and HP1 $\beta$ was also detected. Both Parps selectively poly(ADP-ribosylate) HP1 $\alpha$. Using shRNA approaches, we show that Parp-2-dependent poly(ADP-ribosyl)ation is required for primitive-endodermal differentiation possibly by targeting TIF1 $\beta$ to heterochromatic foci, whereas Parp-1 and its activity participate in the maintenance of TIF1 $\beta$-HP1 association required for progression through parietal endodermal differentiation. Both Parps control TIF1 $\beta$-mediated transcriptional activity. In addition, this work identifies Parp-1 and Parp-2 as new actors of the silencing subcode histone that underlies the histone code.

\section{MATERIALS AND METHODS}

\section{Plasmids and antibodies}

Plasmids and antibodies used are detailed in Supplemental Information

\section{Cell culture and establishment of stable depleted cell lines}

Wild-type and mutant F9 cells were grown in Dulbecco's modified Eagle's medium-4.5 g/L glucose (DMEM; Life Technologies, Inc., Gaithersburg, MD, USA) supplemented with $10 \%$ fetal calf serum (FBS; PanBiotech, Aidenbach, Germany) and $1 \%$ Gentamicin (Life Technologies, Inc.) at $37^{\circ} \mathrm{C}$ in $5 \% \mathrm{CO}_{2}$. To induce PrE and PE differentiations, cells were treated, respectively, with $1 \mu \mathrm{M}$ all-trans RA (Sigma, Lyon, France) alone or in combination with $250 \mu \mathrm{M}$ dibutyryl cAMP (dbcAMP; Sigma) as described previously (30). To establish stable scr, shParp-1, and shParp-2 F9 cell lines, $5 \times$ $10^{6}$ exponentially growing F9 cells were transfected with $5 \mu \mathrm{g}$ of XmnI-linearized pSuper-scrParp-2, pSuper-shParp-1, or pSuper-shParp-2 vectors together with $250 \mathrm{ng}$ of AflIII-linearized pGK-Hygro vector. Selection was started by adding 400 $\mu \mathrm{g} / \mathrm{ml}$ Hygromycin B (Roche, Basel, Switzerland) to the growth medium $24 \mathrm{~h}$ posttransfections for over a period of 2 wk. Several drug-resistant colonies were isolated, expanded, and analyzed for the absolute levels of Parp-1, Parp-2, TIF1 $\beta$, HP $1 \alpha$, and actin by Western blotting. To establish stable shParp-1;shParp-2 cell lines, $5 \times 10^{6}$ exponentially growing cells of a selected shParp-1 F9 clone were transfected with 5 $\mu \mathrm{g}$ of XmnI-linearized pSuper-shParp-2 together with $250 \mathrm{ng}$ of AflIII-linearized pGK-Neo plasmid. Selection was started by adding $600 \mu \mathrm{g} / \mathrm{ml}$ neomycin to the growth medium $24 \mathrm{~h}$ posttransfections for over a period of 2 wk. Neomycinresistant clones were isolated, expanded, and analyzed as mentioned above. Immunoprecipitation, mass spectrometry, Western blot
analysis, and glutathione $S$-transferase (GST) pull-down

For immunoprecipitation in testis cells, 30 testes were collected from 10-wk-old C57/Bl6 mice (Janvier, Le Genest Saint Isle, France) and homogenized by 20 Dounce (no. 2) strokes in lysis buffer $[10 \mathrm{mM}$ Tris-HCl, $\mathrm{pH} 8 ; 400 \mathrm{mM} \mathrm{NaCl} ; 1 \%$ Nonidet P-40; $2 \mathrm{mM}$ dithiothreitol (DTT); $0.5 \mathrm{mM}$ Pefabloc, and protease inhibitor complex (PIC; Roche]. Cell lysates were incubated on ice for $30 \mathrm{~min}$, then digested with 300 $\mathrm{U} / \mathrm{ml}$ of DNase I at $25^{\circ} \mathrm{C}$ for $30 \mathrm{~min}$. After centrifugation at $15,000 \mathrm{rpm}$ at $4^{\circ} \mathrm{C}$ for $20 \mathrm{~min}$, cleared suspension was 
quantified by Bradford protein assay. Proteins (10 mg) were incubated overnight at $4^{\circ} \mathrm{C}$ with either purified anti-Parp-2 pAb or rabbit anti-mouse antibody as control, followed by $2 \mathrm{~h}$ incubation at $4^{\circ} \mathrm{C}$ with protein A sepharose (GE Healthcare, Little Chalfont, UK). The immunoprecipitates were washed twice with washing buffer $(10 \mathrm{mM}$ Tris- $\mathrm{HCl}, \mathrm{pH} 8 ; 0,1 \%$ Nonidet P-40; $2 \mathrm{mM}$ DTT; $0.5 \mathrm{mM}$ Pefabloc; and PIC) containing $500 \mathrm{mM} \mathrm{NaCl}$ and twice with washing buffer containing $50 \mathrm{mM} \mathrm{NaCl}$. Final pellets were resuspended in 50 $\mu \mathrm{l}$ of Laemmli buffer and subjected to $10 \%$ SDS-PAGE. Coprecipitated proteins were stained by SyproRuby (Molecular Probes, Eugene, OR, USA) according to the manufacturer's instructions. Nano-liquid chromatography-mass spectrometry/mass spectrometry experiments were performed using a CapLC capillary liquid chromatography system (Waters, Milford, MA, USA) coupled to a hybrid quadrupole time-of-flight mass spectrometer (Q-TOF II, Waters) according to standard protocols (31). For immunoprecipitation in F9 or F9-derived cells, $10^{7}$ cells were lysed by 3 cycles of freezing and thawing in lysis buffer as above. Cleared lysates were quantified by Bradford protein assay. Following treatment with RNase I (1 $\mathrm{mg} / \mathrm{ml}$ ) for $30 \mathrm{~min}$ at room temperature, $200 \mu \mathrm{g}$ of total proteins was incubated with purified anti-Parp-2 pAb, antiTIF1 $\beta$ mAb, or the control antibody overnight at $4^{\circ} \mathrm{C}$ and immunoprecipitated using protein A sepharose for $2 \mathrm{~h}$ at $4^{\circ} \mathrm{C}$. Beads were washed with $(10 \mathrm{mM}$ Tris $\mathrm{HCl}, \mathrm{pH} 8 ; 50$ to 500 $\mathrm{mM} \mathrm{NaCl} ; 0.1 \%$ Nonidet P-40; 2 mM DTT, and PIC), resuspended in Laemmli buffer, and analyzed by $10 \%$ SDS-PAGE and immunoblotting. Blots were probed with the appropriate specific antibodies followed by peroxidase-conjugated secondary antibodies, and developed using the $\mathrm{ECL}^{+}$detection kit (Amersham, Little Chalfont, UK). When indicated, 100 nM of the Parp inhibitor Ku-0058948 (32) was added to the culture medium $2 \mathrm{~h}$ before lysis and maintained throughout the experiment. GST pull-down analysis was performed as described previously (22).

\section{Immunofluorescence}

Immunofluorescence was performed as described previously (25). Images were captured using a Leica microscope (Leica Microsystems, Heidelberg, Germany) and the capture softAQ: 4 ware OpenLab.

\section{In vitro binding assays}

Escherichia coli expression and purification of GST, GSTTIF1 $\beta$, and GST-HP1 fusion proteins were performed as described previously (10). Equivalent amounts of purified proteins, quantified by Coomassie staining after SDS-PAGE, were incubated with $300 \mathrm{ng}$ of either purified human Parp-1 or murine Parp-2 in binding buffer (20 mM Tris-HCl, pH 7.5; $300 \mathrm{mM} \mathrm{NaCl}$; $0.5 \mathrm{mM}$ Pefabloc; 0.1\% Nonidet P-40; and PIC) for $2 \mathrm{~h}$ at $4^{\circ} \mathrm{C}$. The beads were washed twice with washing buffer $(10 \mathrm{mM}$ Tris- $\mathrm{HCl}, \mathrm{pH} 8 ; 2 \mathrm{mM}$ DTT; $0.5 \%$ Nonidet P-40; $0.5 \mathrm{mM}$ Pefabloc; and PIC) containing $500 \mathrm{mM} \mathrm{NaCl}$ and twice in washing buffer containing $50 \mathrm{mM} \mathrm{NaCl}$. Beads were resuspended in Laemmli buffer and analyzed by Western-blotting.

\section{Heteromodification and noncovalent binding of poly(ADP-ribose) on GST-fusion proteins}

E. coli-expressed GST fused proteins were purified as above and quantified by Coomassie staining on SDS-PAGE. Heteromodification of equivalent amounts of purified proteins by either human Parp-1 or mouse Parp-2 was performed as described previously (33).
For noncovalent binding, ${ }^{32} \mathrm{P}$-labeled poly(ADP-ribose) was synthesized and purified according to Dantzer et al. (22). Equivalent amounts of purified E. coli expressed GST-fused proteins and $1 \mu \mathrm{g}$ of purified recombinant XRCC1 were spotted directly onto nitrocellulose and incubated with ${ }^{32} \mathrm{P}$ labeled poly(ADP-ribose) as described previously (22).

\section{Quantitative RT-PCR}

Quantitative reverse transcriptase-polymerase chain reaction (RT-PCR) using total F9 RNA samples was performed by using the Quantitect SYBR Green PCR kit (Qiagen, Valencia, CA, USA) following the manufacturer's instructions in combination with the Light Cycler Detection System. The PCR products were analyzed with the manufacturer's software. The following primer sequences were used: Hprt-5' $5^{\prime} 5^{\prime}$-TGACACTGGCAAAACAATGCA-3'; Hprt-3' , 5'-GGTCCTTTTCACCAGCAAGCT-3'; Mest-5', 5'-CTCCAAAAACTCTGGATACG-3'; Mest-3', 5'-GAAATTCAGAAGACGCTGGG-3'; HNF4-5', ACACGTCCCCATCTGAAGGTG; HNF4-3', CTTCCTTCTTCATGCCAGCCC.

\section{RESULTS}

Association of Parp-2 and Parp-1 with TIF1及 and $H P 1 \alpha$ in mammalian cells

To identify interacting partners of Parp-2, testis cell extracts were immunoprecipitated with an anti-Parp-2 antibody or a control antibody, and captured proteins were analyzed by mass spectrometry (Fig. 1A). Among the proteins identified, we isolated previously described partners of Parp-2, including Parp-1 and DNA polymerase $\beta$ (33) and a partner of Parp-1 named macroH2A1.2 (34), thus clearly supporting the validity of the approach used. Interestingly, we isolated 6 unique tryptic peptides from TIF1 $\beta$. The functional similarities between Parp-2, Parp-1, and TIF1 $\beta$, with regard to their accumulation on pericentric $\mathrm{HC}$ and their role in differentiation pathways, in addition to the previously described interaction of TIF $1 \beta$ with HP $1 \alpha$ in mouse embryocarcinoma F9 cells, prompted us to investigate further whether these proteins could be physically associated in this model (Fig. $1 B$ ). F9 cell extracts were immunoprecipitated with an anti-Parp-2 antibody or an irrelevant antibody, and coimmunoprecipitation of TIF1 $\beta$ and HP1 $\alpha$ was assessed by Western blotting. We detected significant association of TIF1 $\beta$ and HP1 $\alpha$ with Parp-2 in F9 cells (Fig. $1 B$, lane 2), whereas no association was detected using the control antibody (Fig. 1B, lane 1). In an analog experiment, when F9 cell extracts were immunoprecipitated with an anti-TIF1 $\beta$ antibody or a control antibody, significant fractions of HP1 $\alpha$ and Parp-1 were found in the TIF $1 \beta$ immunoprecipitate (Fig. 1B, lane 6) but not in the control immunoprecipitate (Fig. 1B, lane 5). Taken together, these results describe an association of a subset of Parp-1 and Parp-2 with TIF1 $\beta$ and HP1 $\alpha$ in mammalian cells. 
A

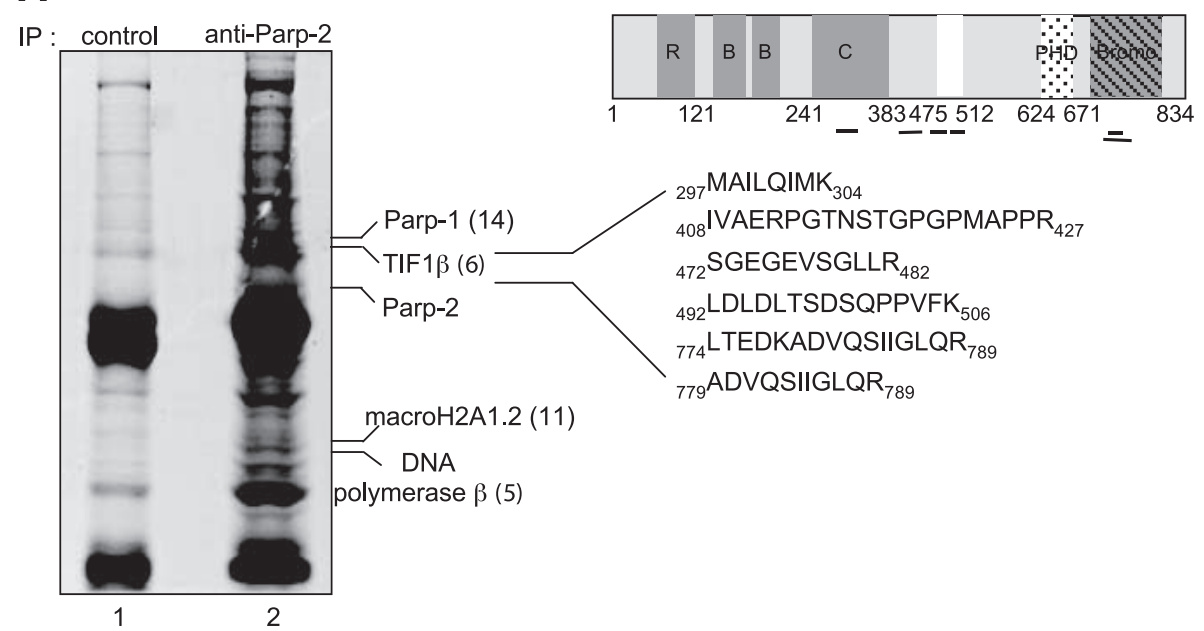

B
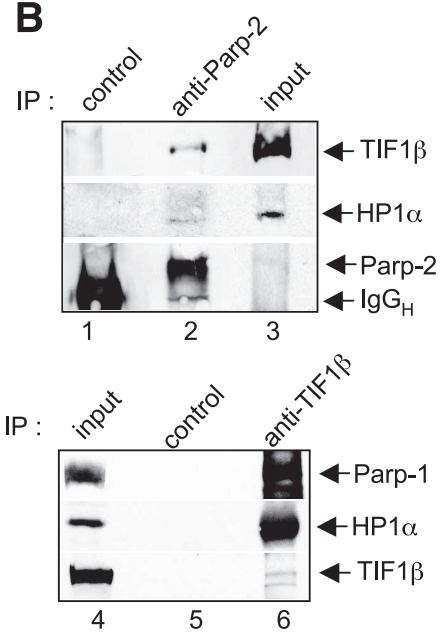

Figure 1. Association of Parp-1 and Parp-2 with TIF1 $\beta$ and HP1 $\alpha$ in mammalian cells. A) Identification of Parp-2-associated TIF1 $\beta$ by mass spectrometry. Testis cell extracts were immunoprecipitated using a control antibody (lane 1) or an anti-Parp- 2 antibody (lane 2). Interacting proteins were resolved by SDS-PAGE, stained with Sypro-Red, and analyzed by mass spectrometry. Numbers in brackets refer to the number of peptides matched. Six different peptides (sequences shown) of TIF1 $\beta$ were found localized along the sequence. B) Left panel: coimmunoprecipitation of TIF1 $\beta$ and HP1 $\alpha$ with Parp-2 in F9 cells. F9 cell extracts were immunoprecipitated with a control antibody (lane 1) or an anti-Parp-2 antibody (lane 2) and analyzed by Western blotting using successively anti-TIF1 $\beta$, anti-HP1 $\alpha$, and anti-Parp-2 antibodies. Input corresponds to $1 / 20$ of the amount of cell extract used for immunoprecipitation (lane 3). Right panel: coimmunoprecipitation of Parp-1 and HP1 $\alpha$ with TIF1 $\beta$ in F9 cells. F9 cell extracts were immunoprecipitated with an anti-TIF1 $\beta$ antibody (lane 6) or a control antibody (lane 5) and analyzed by Western blot. For detection of TIF1 $\beta$ and HP $1 \alpha, 1 / 8$ of the immunoprecipitate was probed using successively anti-HP1 $\alpha$ and anti-TIF $1 \beta$ antibodies. For detection of Parp-1, 7/8 of the immunoprecipitate was probed using an anti-Parp-1 antibody. Lane 4 (input) corresponds to $1 / 25$ the amount of cell extract used for immunoprecipitation.

\section{Parp-2 and Parp-1 interact differentially with HP1 isoforms and TIF1 $\beta$ in vitro}

To characterize the complex further, binding assays between Parp-1, Parp-2, TIF1 $\beta$, and HP1 $\alpha$ were performed in vitro using purified recombinant proteins (Fig. 2). To carefully address the specificity of the interaction with members of the HP1 family, we also tested HP1 $\beta$ and HP1 $\gamma$. GST-HP1 fusion proteins were expressed in E. coli, purified on glutathione $S$-sepharose beads, and batched with purified recombinant Parp-1 or Parp-2. After GST-pull down followed by stringent washes, copurification of Parps were analyzed by Western blotting. As shown in Fig. $2 A$ (top panel), we detected binding of Parp-2 to GST-HP1 $\alpha$ (lane 4) and

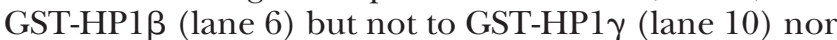
GST (lanes 2, 8). In contrast, Parp-1 bound weakly but reproducibly to GST-HP1 $\beta$ only (lane 5).

To identify the region of HP1 $\alpha$ to which Parp-2 binds, purified GST fusion proteins expressing various HP1 $\alpha$ deletion domains-GST-CD, GST-hinge domain, and GST-CSD-were tested for interaction with purified Parp-2. As shown in Fig. $2 A$ (bottom panel), Parp-2 interacts with the CSD (lane 3) and hinge domain (lane 2) of $\mathrm{HP} 1 \alpha$, whereas no interaction was detected with the CD (lane 1). Thus Parp-2 but not Parp-1 can directly interact with $\mathrm{HP} 1 \alpha$ in vitro.

Because TIF $1 \beta$ also interacts directly with HP1 $\alpha$ (11), we next addressed whether the association of TIF1 $\beta$ with Parp-2 detected in F9 cells (Fig. $1 B$ ) requires HP1 $\alpha$. Flag-tagged proteins expressing either wild-type TIF1 $\beta$
(Flag-TIF1 $\beta$ ) or TIF1 $\beta$ with a mutation in the HP1 box that disrupts its interaction with HP1 $\alpha$ (Flag-TIF1 $\beta^{\text {HPlbox }}$ ) were coexpressed in Cos1 cells together with GST-Parp-2 or GST alone. To test the role of poly(ADP-ribosyl)ation, the same assay was performed in the presence of the Parp inhibitor $\mathrm{Ku}-0058948$. Coprecipitating proteins were analyzed by GST pull-down experiments and Western blotting. As shown in Fig. $2 B$, we observed a significantly weaker copurification of Flag-TIF1 $\beta^{\text {HPlbox }}$ with GSTParp-2 (lane 4) when compared to Flag-TIF1 $\beta$ (lane 3). Thus, the HP1 box mutation of TIF1 $\beta$ that was previously described to disrupt the interaction between TIF1 $\beta$ and HP1 proteins (19) also impairs the interaction between TIF1 $\beta$ and Parp-2, which suggests that TIF1 $\beta$-Parp-2 association involves-at least partly-HP1 proteins. No copurification with GST was detected (Fig. 2B, lane 2). The addition of Ku-0058948 also significantly impaired the binding of Flag-TIF1 $\beta$ to GST-Parp-2, thus indicating a role of poly(ADP-ribose) in the association of both partners (Fig. 2B, lanes 1,3).

We next compared the direct binding efficiency of Parp-1 and Parp-2 to TIF1 $\beta$ (Fig. 2C). Under similar conditions of binding assays as above, we identified an efficient binding of Parp-2 to TIF1 $\beta$ and a weaker but reproducible interaction with Parp-1 (Fig. 2C, left panel). To verify whether Parp-2 and TIF1 $\beta$ also associate independently of HP1 in vivo, nuclear extracts from TIF $1 \beta^{\text {HP1box/- }}$ cells expressing the mutated TIF $1 \beta^{\text {HPlbox }}$ were immunoprecipitated with an anti-Parp-2 antibody, and the immunoprecipitates were probed for the presence of TIF1 $\beta$ (Fig. 2C, right panel). Coimmunoprecipi- 
A

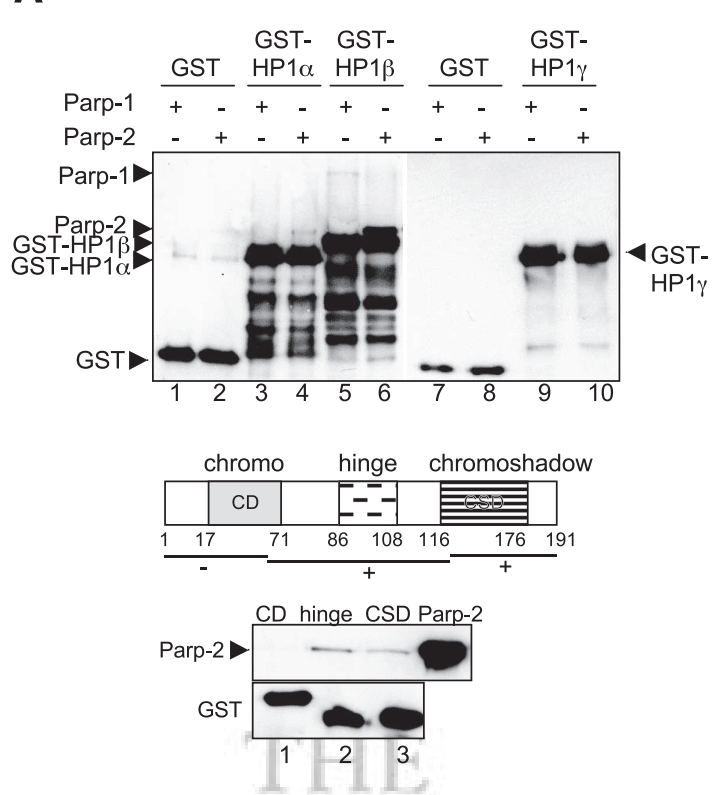

B

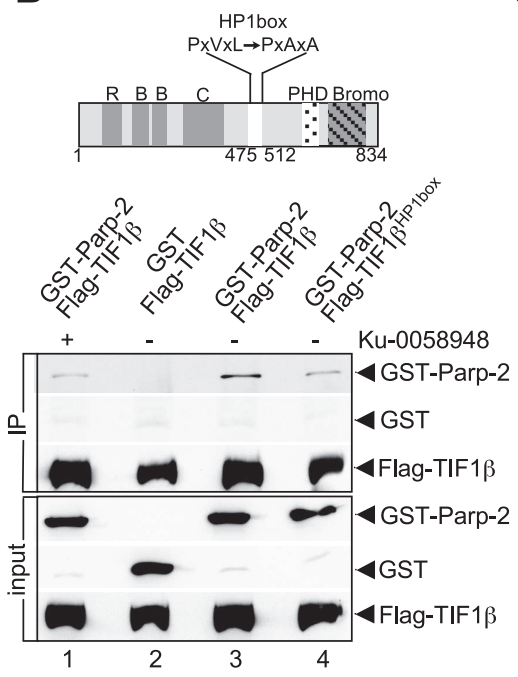

C

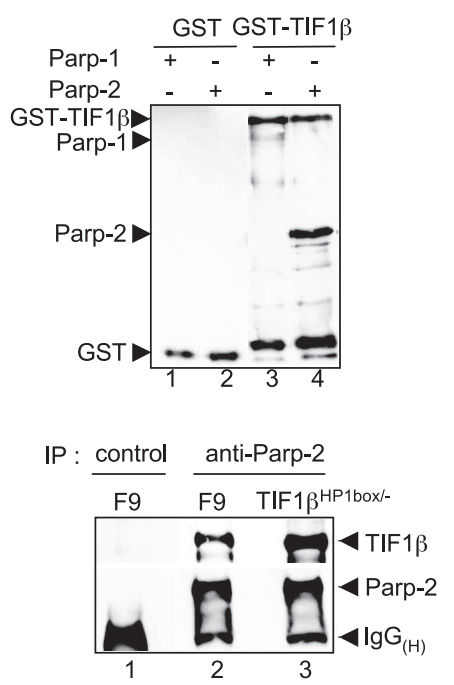

Figure 2. Differential interaction of Parp-2 and Parp-1 with HP1 isoforms and TIF1 $\beta$. A) In vitro interaction of Parp-1 and Parp-2 with HP1 isotypes. Top panel: purified recombinant Parp-1 (lanes 1, 3, 5, 7, 9) or Parp-2 (lanes 2, 4, 6, 8, 10) was incubated in a batch assay with purified GST (lanes 1, 2 and 7, 8), GST-HP1 $\alpha$ (lanes 3, 4), GST-HP1 $\beta$ (lanes 5, 6) or GST-HP1 $\gamma$ (lanes 9, 10). Bound Parps were analyzed by GST pull-down and Western blotting using successively anti-Parp-1, anti-Parp-2, and anti-GST antibodies. Bottom panel: purified recombinant Parp-2 was incubated in a batch assay with purified GST-fused CD (GST-CD ${ }_{1-66}$, lane 1), GST-fused hinge domain (GST-hinge ${ }_{67-119}$, lane 2) or GST-fused CSD (GST-CSD 119-191 $_{1}$, lane 3) of HP1 $\alpha$. Bound Parp-2 proteins were analyzed by GST pull-down and Western blotting using successively anti-Parp-2 and anti-GST antibodies. $B$ ) The HP1 box is required for the association of TIF1 $\beta$ with Parp-2 in F9 cells. Top panel: schematic representation of TIF1 $\beta$ indicating the TIF1 $\beta^{\text {HP1box }}$ mutant used. Bottom panel: lysates from Cos1 cells expressing GST-Parp-2 (lanes 1, 3, 4) or GST (lane 2) together with either Flag-TIF1 $\beta$ (lanes 1-3) or Flag-TIF1 $\beta^{\text {HP1box }}$ (lane 4) were analyzed by GST pull-down and Western blotting using anti-TIF1 $\beta$ and anti-GST antibodies, respectively. In lane 1, Ku-0058948 was added throughout the experiment. C) Parp-2 directly interacts with TIF1 $\beta$. Left panel: purified recombinant Parp-1 (lanes 1-3) or Parp-2 (lanes 2, 4) was incubated in a batch assay with purified GST or GST-TIF1 $\beta$. Bound Parps were analyzed by GST pull-down and Western blotting using successively anti-Parp-1, anti-Parp-2, and anti-GST antibodies. Right panel: wild-type F9 (lanes 1, 2) and mutant TIF1 $\beta^{\mathrm{HP} 1 \mathrm{box} /-}$ (lane 3 ) cell extracts were immunoprecipitated with a control antibody (lane 1) or an anti-Parp-2 antibody (lanes 2, 3) and analyzed by Western blotting using successively anti-TIF1 $\beta$ and anti-Parp-2 antibodies. HIE LIFE SCIFNCES

tation of TIF1 $\beta$ with Parp-2 was detected both in TIF1 $\beta^{\text {HP1box/- }}$ cells (Fig. 2C, lane 3) and in the parental F9 cells (Fig. 2C, lane 2), but not in control immunoprecipitate (Fig. 2C, lane 1).

Finally, we found that TIF1 $\beta$ interacts with either the central $\mathrm{E}$ or the catalytic $\mathrm{F}$ domains of Parp-2 but not the N-terminal DNA-binding domain (data not shown).

Taken together, these results describe an heterochromatic protein network characterized by 1) a selective efficient binding of Parp-2 to HP1 $\alpha$ and HP1 $\beta$ but not HP1 $\gamma$, 2) an association of Parp-2 with TIF1 $\beta$ both directly and through HP1, and 3) a weaker but reproducible direct binding of Parp-1 to HP1 $\beta$ and TIF1 $\beta$.

\section{Selective poly(ADP-ribosyl)ation and noncovalent binding of poly(ADP-ribose) to HP1 $\alpha$}

To gain further insights into the functional interactions governing this protein network, we evaluated the ability of either Parp-1 or Parp-2 to poly(ADP-ribosyl)ate HP1 GST-TIF1 $\beta$ fusion proteins or GST alone were incubated with either Parp-1 or Parp-2 or no protein, in the presence of $\alpha-{ }^{32} \mathrm{PNAD}^{+}$and DNase-I-treated calf thy-

mus DNA. Autoradiography revealed that both Parp-1 and Parp-2 were able to poly(ADP-ribosyl) ate selectively HP1 $\alpha$ but not HP1 $\beta$, HP1 $\gamma$, TIF1 $\beta$, or GST alone (Fig. $3 A)$. To identify the domain of $\mathrm{HP} 1 \alpha$ poly(ADP-ribosyl)ated, we used the same approach with the purified $E$. coli-expressed deletion domains of HP1 $\alpha$ and found that $\mathrm{HP} 1 \alpha$ is poly(ADP-ribosyl)ated on its hinge domain by both Parp-1 and Parp-2 (Fig. 3B).

To test whether HP1 isoforms or TIF1 $\beta$ could directly bind to poly(ADP-ribose), similar amounts of purified GST, GST-HP1, and GST-TIF1 $\beta$ fusion proteins were spotted onto nitrocellulose and incubated with radioactive poly(ADP-ribose) (Fig. 3C). Detection of a radioactive signal was observed only for GST-HP1 $\alpha$, thus showing that HP1 $\alpha$ binds tightly and stably to the poly(ADP-ribose). The same conditions were used to identify the domain of HP1 $\alpha$ that could bind to poly(ADP-ribose) and showed that PAR binds specifically to the hinge domain (Fig. 3D).

\section{Interaction and colocalization of Parp-2 with TIF1 $\beta$ onto pericentric $\mathrm{HC}$ in $\mathrm{PrE}$ cells}

The above results and the recent advances describing an essential role of TIF1 $\beta-\mathrm{HP} 1$ interaction during 
A

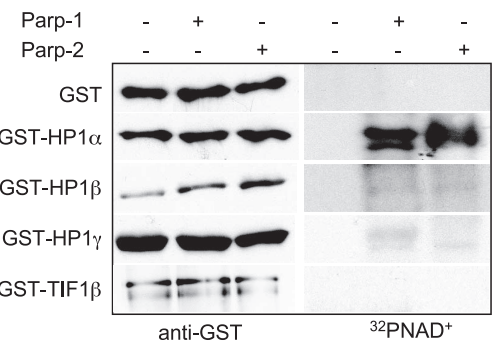

D

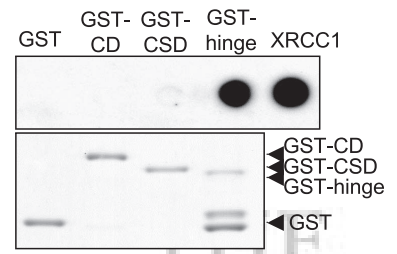

B
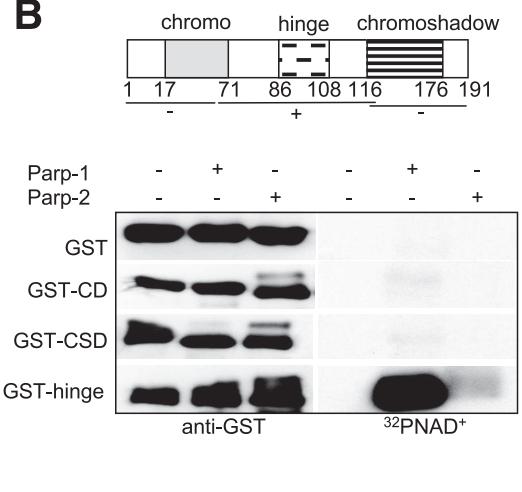

GST- GST- GST- GST

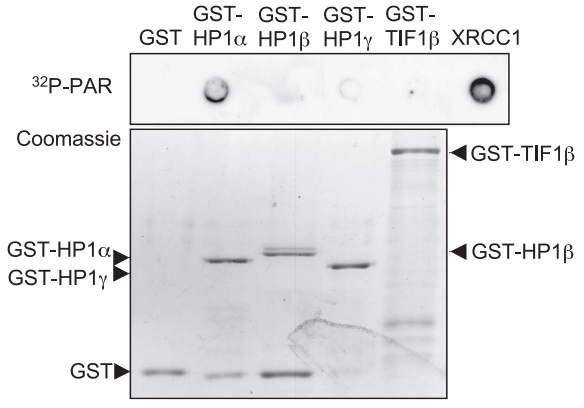

Figure 3. The hinge domain of $\mathrm{HP} 1 \alpha$ is poly(ADP-ribosyl)ated and binds poly(ADPribose). $A, B)$ The hinge domain of HP1 $\alpha$ is poly(ADP-ribosyl)ated by Parp-1 and Parp-2. A) Purified GST, GST-HP1 $\alpha$, GST-HP1 $\beta$, GST-HP1 $\gamma$, and GST-TIF1 $\beta$ were incubated with either Parp-1 or Parp-2 in activity buffer containing $\left[\alpha_{-}{ }^{32} \mathrm{P}\right] \mathrm{NAD}^{+}$and DNase I-activated DNA. Right panel: autoradiography. Left panel: fusion proteins were analyzed by Western blotting with an anti-GST antibody. B) Top panel: schematic representation of HP1. Bottom panel: similar experiment was performed as in $A$, using purified GST, GST-CD ${ }_{1-66}$, GST-CSD $_{119-191}$ or GST-hinge ${ }_{67-119}$ domains of HP1 $\alpha$. Right panel: autoradiography. Left panel: fusion proteins analyzed by Western blotting using an anti-GST antibody. $C$, $D$ ) Poly(ADP-ribose) binds to the hinge domain of HP1 $\alpha$. C) Purified GST, GST-HP1 $\alpha$, GST-HP1 $\beta$, GST-HP1 $\gamma$, and GST-TIF1 $\beta$ were spotted on nitrocellulose and incubated with $\left[{ }^{32} \mathrm{P}\right]$ poly(ADP-ribose). Top panel: autoradiography. Bottom panel: the amount of protein loaded was controlled by SDS-PAGE and Coomassie staining. $D$ ) Similar experiment was performed as in $C$,

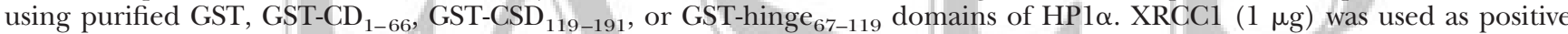
control (22). Top panel: autoradiography. Bottom panel: the amount of protein loaded was controlled by SDS-PAGE and Coomassie staining.

endodermal differentiation (19) prompted us to follow the association of Parps and TIF1 $\beta$ in this process. We first analyzed the expression of Parp-1, Parp-2, TIF1 $\beta$, and HP1 $\alpha$ in nuclear extracts of undifferentiated F9 or differentiated PrE and PE cell lines. Whereas the expression of Parp-1 and TIF1 $\beta$ decreased throughout differentiation of F9 to PrE and PE cells, the expression of Parp-2 and HP1 $\alpha$ remained constant (Fig. 4A).

We next monitored the colocalization of Parp- 1 and Parp-2 with TIF1 $\beta$ throughout the differentiation of F9 cells by indirect immunofluorescence (Fig. 4B). As described previously, Parp-2 (Fig. 4Bb) and Parp-1 (Fig. $4 B k$ ) displayed a nuclear punctate distribution in F9 cells, with, however, a stronger accumulation in nucleoli (25), whereas TIF1 $\beta$ (Fig. $4 B a, j$ ) showed homogeneous nuclear staining and was excluded from nucleoli. Interestingly, the differentiation into PrE induced a dynamic targeting of Parp-2 (Fig. $4 B e$ ) and TIF1 $\beta$ (Fig. $4 B d, m$ ) but not Parp-1 (Fig. $4 B n$ ) onto pericentric HC foci where both proteins colocalize. On further differentiation into PE cells, Parp-1 (Fig. 4Bq), Parp-2 (Fig. $4 B h$ ), and TIF1 $\beta$ (Fig. $4 B g, p$ ) exhibited a pattern similar to undifferentiated cells, although with less accumulation of Parps in the nucleoli.

To support this result further, we examined the association of Parp-2 and TIF1 $\beta$ throughout differentiation. Nuclear extracts of F9, PrE, and PE cells were immunoprecipitated with an anti-Parp-2 antibody or an irrelevant antibody, and the immunoprecipitates were probed for the presence of TIF1 $\beta$ (Fig. 4C). The amount of TIF1 $\beta$ coimmunoprecipitated was correlated with the expression of each partner throughout differentiation. Indeed, TIF1 $\beta$ was clearly detected in Parp-2 immunoprecipitates of F9 and PrE cell extracts (Fig. 4C, lanes 2,3) containing significant amounts of proteins, but not readily in $\mathrm{PE}$ cell extracts, due to limited amounts of proteins expressed (Fig. 4C, lane 4). No TIF $1 \beta$ was detected in the control immunoprecipitate (Fig. $4 C$, lane 1). Together, these results indicate that the interaction of TIF1 $\beta$ with Parp-2 is maintained in the differentiated PrE cells.

Altogether, these data indicate that Parp-2 associates with TIF1 $\beta$ within regions of pericentric HC throughout differentiation of F9 to PrE cells in addition to the protein complex formed in the euchromatin compartment of F9 stem cells (Fig. 1).

\section{Parp-2 is required for the differentiation into PrE cells, whereas Parp-1 is required for terminal differentiation into $\mathrm{PE}$ and visceral-endoderm-like (VE) cells}

To investigate whether the absence of either Parp-2, Parp-1, or both lead to a defect in the differentiation of F9 cells to endoderm-like cells, we used the shRNA approach to generate stable clones depleted in either Parp-1 (shParp-1), Parp-2 (shParp-2), or both proteins (shParp-1;shParp-2). A Western blot analysis of the selected clones is shown in Fig. 5A. When compared to the expression of a housekeeping protein $\beta$-actin, the extent of Parps depletion was estimated to be more than $99 \%$ in the selected clones. Noticeably, the depletion of either Parp-1, Parp-2, or both had no effect on the level of TIF1 $\beta$ expression. 
A

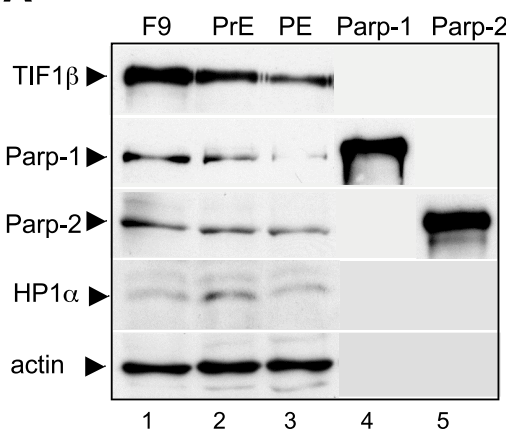

C

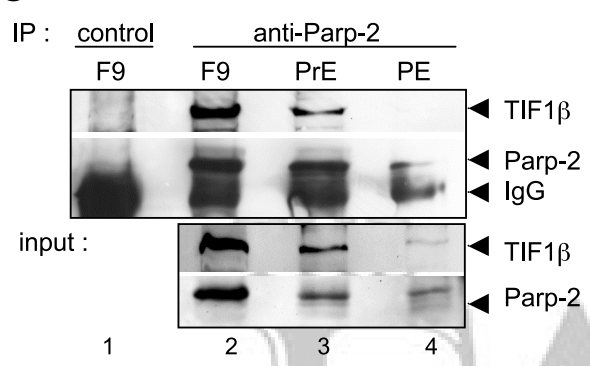

B

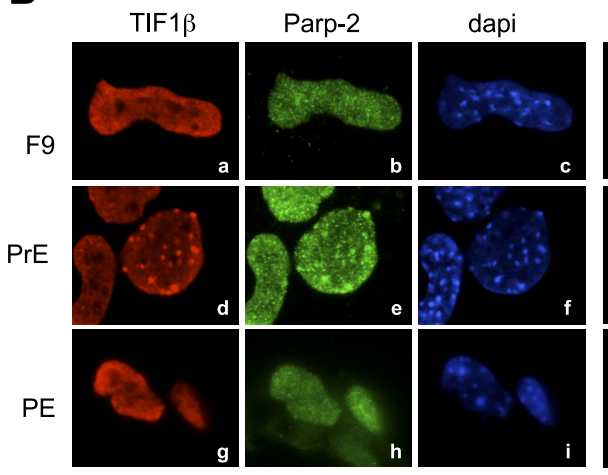

Parp-1

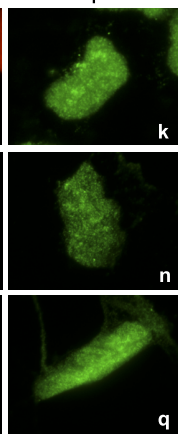

dapi

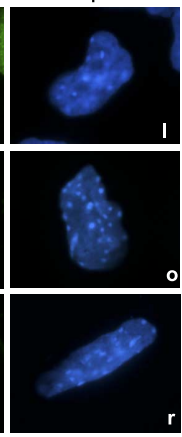

Figure 4. Parp-2 interacts and colocalizes with TIF1 $\beta$ in PrE cells. A) Expression of Parp-1, Parp-2, TIF1 $\beta$, and HP1 $\alpha$ throughout differentiation. F9 cells (lane 1) were induced to differentiate into PrE (lane 2) or PE cells (lane 3). Equivalent amounts of total protein extracts were separated by SDS-PAGE and analyzed by Western blotting with the appropriate antibodies. Lane 4: purified recombinant Parp-1 (50 ng). Lane 5: purified recombinant Parp-2 (50 ng). B) Left panel: colocalization of Parp-2 and TIF1 $\beta$ to centromeric HC in PrE cells. F9 cells were induced to differentiate into PrE and PE cells and stained red with Alexa 568-labeled mouse anti-TIF1 $\beta$ antibody $(a, d, g)$ and green with Alexa 488-labeled rabbit anti-Parp-2 antibody $(b, e, h)$. DNA was couterstained with $4^{\prime}, 6^{\prime}$-diamidino-2-phenylindole (dapi) $(c, f, i)$. Right panel: subcellular localization of Parp-1 throughout differentiation. F9 cells were induced to diffentiate into PrE and PE cells and stained red with Alexa 568 -labeled mouse anti-TIF1 $\beta$ antibody $(j, m, p)$ and green with Alexa 488-labeled rabbit anti-Parp-1 antibody $(k, n, q)$. DNA was couterstained with dapi $(l, o, r)$. C) F9 cells were induced to differentiate, and equivalent amounts of total protein cell lysates from F9, PrE, and PE cells were immunoprecipitated with a control antibody (lane 1) or an anti-Parp-2 antibody (lanes 2, 4), and analyzed by Western blotting using successively anti-TIF1 $\beta$ and anti-Parp- 2 antibodies. Input corresponds to $1 / 50$ of the amount of total cell extract used for immunoprecipitation.

We induced each depleted F9 cell line to differentiate and followed differentiation by the morphological features characteristic of PrE and PE cells (30). To evaluate the requirement of Parp-1 or Parp-2 catalytic activity, cells were grown in the absence or in the presence of $\mathrm{Ku}-0058948$ throughout differentiation. As shown in Fig. $5 B$, when grown in the presence of the Parp inhibitor, control scr-F9 cells displayed a weak reduced capacity to differentiate to both PrE (Fig. 5Be) and PE (Fig. 5Bf), which suggests a potential involvement of poly(ADP-ribosyl)ation in both stages. In contrast, the knockdown of Parp-2 clearly impaired differentiation into PrE (Fig. 5Bh), even though a population of remaining cells was still able to progress to PE (Fig. $5 \mathrm{Bi}$ ). The addition of $\mathrm{Ku}-0058948$ had no major additional incidence on the differentiation of shParp-2 to PrE (Fig. 5Bk) but significantly disrupted further differentiation to PE (Fig. 5Bl), thus suggesting a role of Parp-1 catalytic activity in the second stage of differentiation. In line with this observation, Parp-1-depleted cells differentiated into PrE (Fig. 5Bn) but did not differentiate further into PE (Fig. $5 \mathrm{Bo}$ ). The addition of $\mathrm{Ku}-0058948$ significantly impaired the potential of shParp-1 to differentiate to $\operatorname{PrE}$ (Fig. $5 B q$ ), in agreement with an essential role of Parp-2-dependent poly(ADP-ribosyl)ation in primitive endodermal differentiation substantially increased in the absence of Parp-1. As expected, the depletion of both Parp-1 and Parp-2 completely inhibited the differentiation to both $\mathrm{PrE}$ and PE (Fig. $5 B t, u$ ).

Taken together, these data firmly assign an essential role of Parp-2 and its activity in the differentiation of F9 to $\operatorname{PrE}$, whereas Parp- 1 and the associated activity are crucial for terminal differentiation to PE. Expression of Troma-1 in these cells confirmed their differentiated status (data not shown). We also studied the requirement of either Parp-1, Parp-2, or both in an other model of terminal differentiation into VE cells and found an essential role of Parp-1 but not Parp-2 in this process (Supplemental Fig. 1).

Impaired relocation of TIF1 $\beta$ to pericentric $\mathrm{HC}$ in Parp-2-depleted PrE cells but not in Parp-1-depleted PrE cells

Differentiation to PrE is accompanied by relocation of TIF1 $\beta$ from euchromatin to HC (30). To investigate the role of Parp-1 and -2 and poly(ADP-ribosyl)ation in this process, we compared the dynamic relocation of TIF $1 \beta$ onto pericentric HC during PrE differentiation in each stable depleted cell line and in the absence or in the presence of $\mathrm{Ku}-0058948$ (Fig. 6). In nontreated cells, TIF1 $\beta$ staining was homogeneously distributed within the nucleus of scr, shParp-1, shParp-2, or shParp1;shParp-2 F9 cells (data not shown). After 4 days of RA treatment, normal targeting of TIF1 $\beta$ to pericentric HC was observed in an average of 33\% of shParp-1-PrE compared to $31 \%$ in the control scr-PrE, thus indicating that the absence of Parp- 1 has no effect on TIF1 $\beta$ relocation. However, when grown in the presence of $\mathrm{Ku}-0058948$, the percentage of control scr-PrE display- 
A

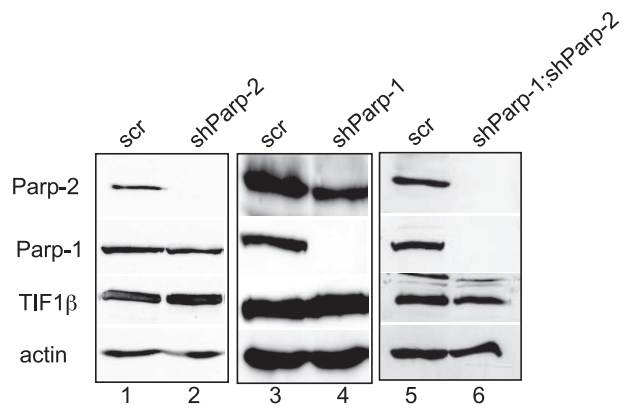

B

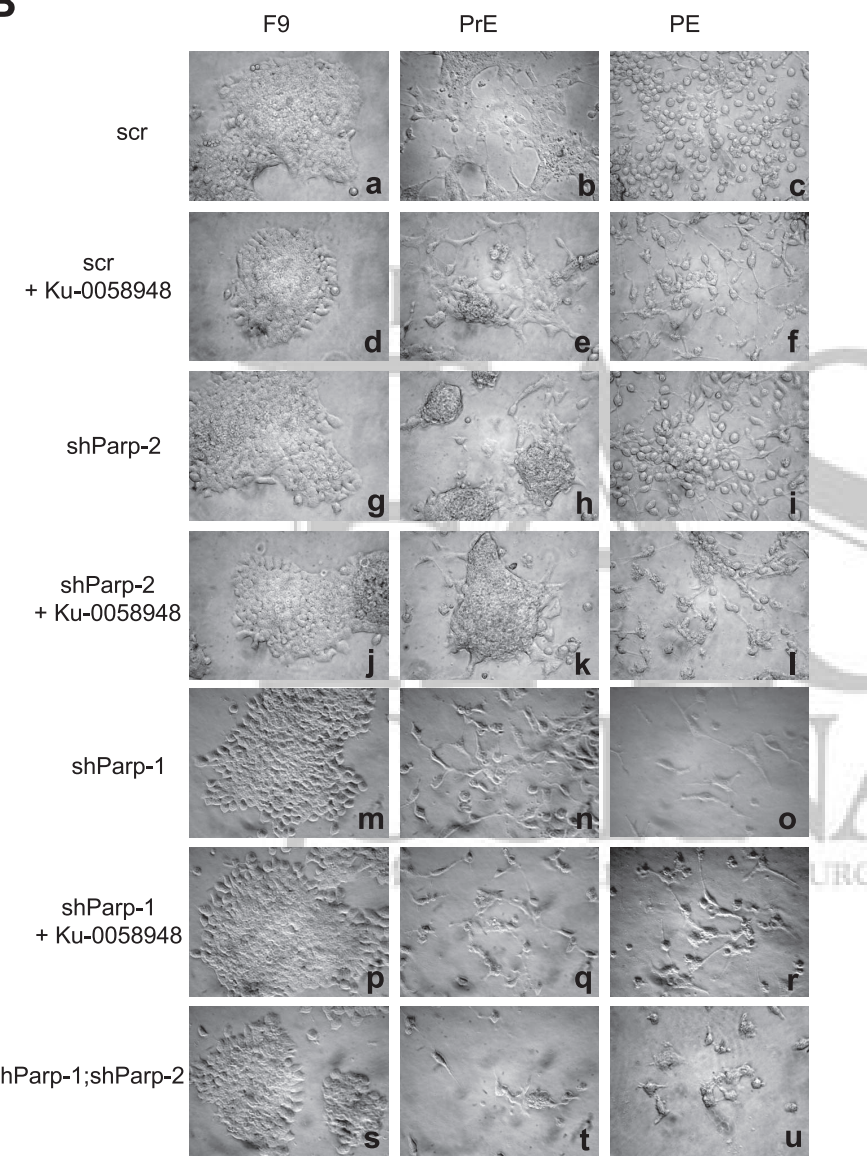

Figure 5. Parp-2-depleted F9 cells are impaired in the differentiation into PrE cells, whereas Parp-1-depleted F9 cells do not differentiate into PE cells. A) Analysis of the extent of depletion of Parps proteins. Western blot analysis for the expression of Parp-2, Parp-1, TIF1 $\beta$, and $\beta$-actin in control (scr) (lanes 1, 3, 5), Parp-2-depleted (shParp-2) (lane 2), Parp-1-depleted (shParp-1) (lane 4), and Parp-1;Parp-2-depleted (shParp-1;shParp-2) (lane 6) F9 cells. B) Effect of Parp-1-, Parp-2-, and Parp-1;Parp-2-depletion on F9 cell differentiation. Scr, shParp-2, shParp-1, and shParp-1;shParp-2 F9 cells were induced to differentiate into PrE, and PE cells as described in Materials and Methods in the absence or in the presence of $\mathrm{Ku}-0058948$ (100 nM) and photographed under a phase contrast microscope.

ing focal staining of TIF1 $\beta$ significantly decreased to $12 \%$, reflecting the involvement of poly(ADP-ribosyl) ation in TIF1 $\beta$ HC targeting. In similar conditions the differentiation to PrE was only slightly affected (Fig.
$5 B e)$. Similarly, the knockdown of Parp-2 clearly impaired TIF1 $\beta$ redistribution to the same extent as for $\mathrm{Ku}$-0058948-treated scr-PrE, thus suggesting an essential role of Parp-2 and its catalytic activity in this process. Accordingly, the addition of Ku-0058948 had no major additional incidence on TIF1 $\beta$ relocation in shParp-2PrE but decreased the percentage of shParp-1-PrE with TIF1 $\beta$ foci.

Taken together, these results reveal an essential specific role of Parp-2, but not Parp-1, and its catalytic activity in the TIF1 $\beta$ targeting to heterochromatic foci during PrE differentiation. Under similar conditions, a wild-type-like accumulation of both $\mathrm{HP} 1 \alpha$ and trimethyl-H3K9 to HC was detected in all cases (Supplemental Fig. 2).

\section{Parp-1 but not Parp-2 controls the interaction between TIF1 $\beta$ and HP1 $\alpha$}

The TIF1 $\beta-H P 1$ interaction is indispensable for $\mathrm{PE}$ differentiation (19). The absence of PE differentiation in shParp-1, shParp-1;shParp-2, or shParp-2 cells treated with $\mathrm{Ku}-0058948$ suggests a role of Parp-1 and its activity in the association of TIF1 $\beta$ with HP1. To test this hypothesis, we examined the association of both partners by coimmunoprecipitation in early differentiating PrE, shParp-1-PrE, and shParp-2-PrE in a window of time in which TIF1 $\beta$-HP1 association is required for terminal differentiation (Fig. $7 A$, left panel). Interestingly, the absence of Parp-1 but not Parp-2 caused a weak but reproducible decrease in the coimmunoprecipitation of HP1 $\alpha$ with TIF1 $\beta$ compared to the association detected in the control F9 (Fig. $7 A$; compare lanes 2, 3 with 1) thus revealing a partial but essential and specific role of Parp-1 compared to Parp-2 in the maintenance of TIF1 $\beta-H P 1 \alpha$ interaction. To examine further the role of poly(ADP-ribosyl)ation in this association, similar coimmunoprecipitation experiments were performed in the presence of the Parp inhibitor (Fig. 7A, right panel). Inhibition of Parp activity also impaired TIF $1 \beta-H P 1 \alpha$ coimmunoprecipitation (Fig. $7 A$; compare lanes 6,7$)$. Taken together, these results are in favor of a role of Parp-1 but not Parp-2 and poly(ADP-ribosyl)ation in the association of TIF1 $\beta$ with HP1 $\alpha$ that controls the progression through terminal differentiation.

\section{Both Parps control TIF1 $\beta$ transcriptional activity}

Gene expression analysis in early differentiating PrE cells has revealed an essential role of TIF1 $\beta-H P 1$ association for induction of the endoderm-specific gene HNF4 (19) and repression of the mesoderm-specific transcript Mest (unpublished data). To investigate the role of Parp-1 and Parp-2 in TIF1 $\beta$ transcriptional activity, we analyzed the transcriptional level of both genes in control (scr), shParp-1, shParp-2, and shParp-1;Parp-2-PrE by qRT-PCR (Fig. $7 B$ ). In agreement with previous data, we detected a 2.4-fold reduction of HNF4 (19) and a 1.72-fold induction of Mest in TIF1 $\beta^{\text {HPlbox/- }}$ cells (data not shown). Surpris- 
A
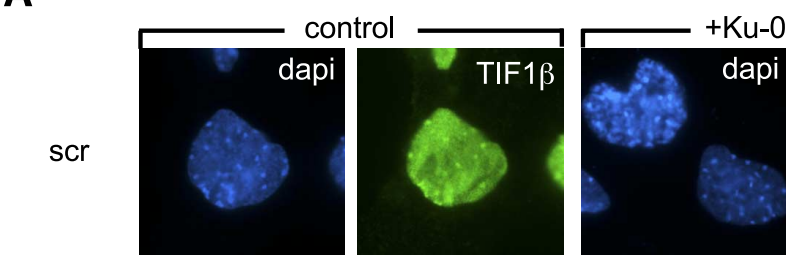

dapi

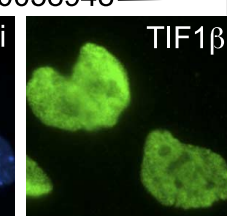

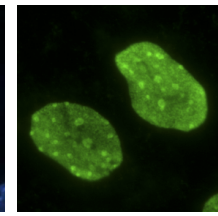

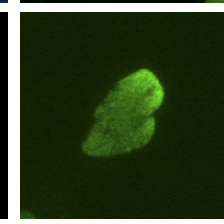

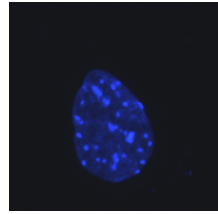

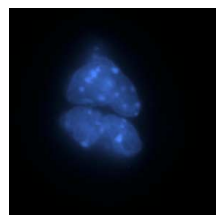

B

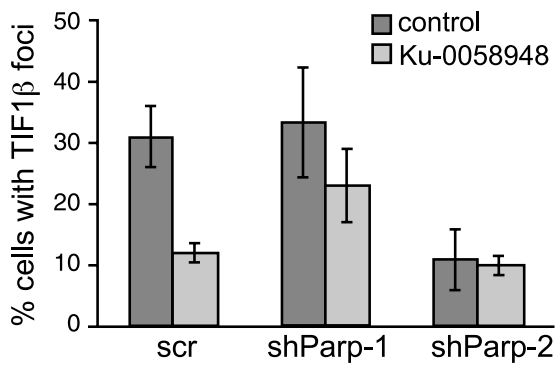

Figure 6. Targeting TIF1 $\beta$ to pericentric HC in PrE cells requires Parp-2 but not Parp-1. A) Scr, shParp-1, and shParp-2 F9 cells were induced to differentiate in PrE in the absence or in the

presence of Ku-0058948 and processed for immunofluorescence using an anti-TIF1 $\beta$ antibody (green). DNA was counterstained with dapi (blue). A representative cell according to the histogram in $B$ is shown. $B$ ) Histogram showing percentage of cells displaying TIF1 $\beta$ heterochromatic foci. Cells in $>20$ randomly selected immunofluorescence fields were scored for the presence or absence of TIF1 $\beta$ nuclear foci. An average of 500 cells was scored per cell line and condition. Results are averages from at least 3 independent experiments.

ingly, whereas the expression of HNF4 was equivalent in control and shParp-1 cells, we found a significant reduced expression in shParp-2 and shParp-1;Parp-2 cells in comparison with control cells (Fig. 7B, left panel; 8- and 3.2-fold, respectively). In contrast, Mest was up-regulated by 5.9-, 7-, and 3.9-fold in the absence of either Parp-1, Parp-2, or both Parps, respectively (Fig. 7B, right panel). Together, these results clearly indicate a role of both Parps in the expression of TIF1 $\beta$-regulated specific genes in addition to the critical role of TIF1 $\beta-H P 1$ complex.

\section{DISCUSSION}

It was previously shown that TIF1 $\beta$-HP1 interaction plays a critical role during F9 cell differentiation (19).
Here, we provide evidence for physical and functional selective interactions between Parp-1, Parp-2, TIF1 $\beta$, and HP1 in mammalian cells that provide an additional level of regulation through at least two distinct mechanisms: whereas Parp-2 activity controls TIF1 $\beta$ targeting to pericentric HC, Parp-1 activity essentially acts on TIF1 $\beta$ HP1 association. Both might contribute to TIF1 $\beta$ dependent gene expression.

\section{Differential association of Parp-2 and Parp-1 with TIF1 $\beta$ and HP1 isotypes}

In a proteomic screen of Parp-2 interacting proteins in testis cell extracts, we identified the transcriptional intermediary factor $1 \beta$ (TIF1 $\beta$ ) together with Parp-1.
A

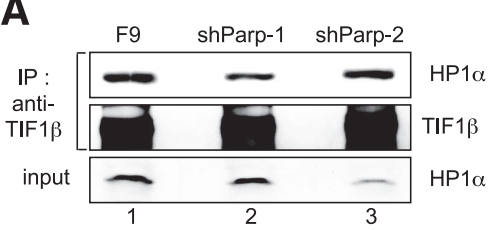

B

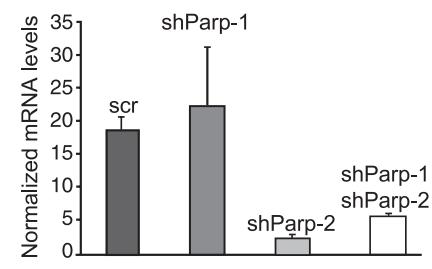

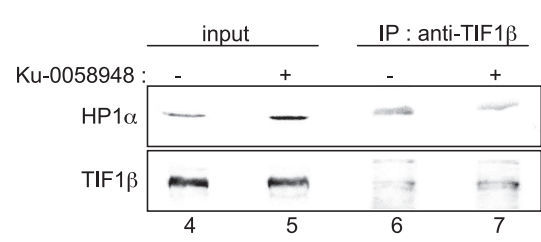

Mest expression :

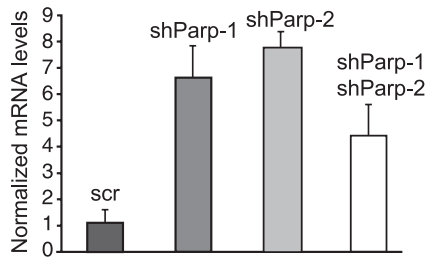

Figure 7. Parp-1 but not Parp-2 is involved in the maintenance of TIF $1 \beta-H P 1 \alpha$ interaction, whereas both proteins control TIF1 $\beta$-dependent transcriptional activity. A) Left panel: F9 (lane 1), shParp-1 (lane 2), and shParp-2 (lane 3) cells were first grown for $24 \mathrm{~h}$ in the presence of RA to induce differentiation. Equivalent amounts of total protein cell lysates from early differentiating cells were then immunoprecipitated using an anti-TIF1 $\beta$ antibody and analyzed by Western blotting using successively anti-HP $1 \alpha$ and antiTIF1 $\beta$ antibodies. Input corresponds to $1 / 6$ of the amount of total cell extract used for immunoprecipitation. Right panel: F9 cells were induced to differentiate for $24 \mathrm{~h}$ in the absence (lanes 4,6 ) or in the presence of $\mathrm{Ku}-0058948$ (lanes 5, 7) and treated for immunoprecipitation and Western-blot analysis as in $A$. B) Quantitative RT-PCR analysis of RNA from scr, shParp-1, shParp-2, or shParp-1;Parp-2 early differentiating cells, for the endoderm-specific transcript HNF4 (left panel) and the mesoderm-specific transcript Mest (right panel) normalized against HPRT control. Histogram displays the ratio of specific gene expression in RA-treated vs. untreated cells. 
Interestingly, both Parps and TIF1 $\beta$ display several functional similarities in support of a dynamic interplay between these partners: 1) both Parps and TIF1 $\beta$ relocate on pericentric $\mathrm{HC}$, in metaphase cells for Parp-1 (23) and Parp-2 (24) and in differentiated cells for TIF1 $\beta$ (30); 2) Parp-1 and Parp-2 (21) and TIF1 $\beta$ (16) exert central cellular functions during embryonic development; and 3) both Parps function in various differentiation processes, including neurogenesis (26), dendritic cell differentiation (35), spermiogenesis (28), adipogenesis (27), and T-lymphocyte development (29), whereas TIF1 $\beta$ in association with HP1 is essential for F9 cells to differentiate into endoderm-like cells (19). In line with this latter observation, we next confirmed that TIF $1 \beta$ and HP $1 \alpha$ can be coimmunoprecipitated with Parp-2 and Parp-1 in F9 cells, thus describing the existence of a protein network involving Parp-1, Parp-2, TIF1 $\beta$, and HP1 $\alpha$. We further characterized this complex combining in vitro protein-protein interaction assays and GST pull-down experiments and show here that both Parp-2 and Parp-1 interact directly but selectively and with significantly different affinities with both HP1 isoforms and TIF1 $\beta$. Whereas Parp-2 binds efficiently and with high affinity to HP1 $\alpha, H P 1 \beta$, and TIF1 $\beta$, Parp-1 interacts only weakly but reproducibly with HP1 $\beta$ and TIF1 $\beta$. The association of Parp-2 with TIF $1 \beta$ is mediated both by a direct interaction of both partners and an indirect interaction through HP1 $\alpha$. Indeed, the PxVxL motif of TIF1 $\beta$ essential for its interaction with HP1 (11) is also required for its association with Parp-2. Together, these data describe Parp- 1 and Parp-2 as new components of the TIF1 $\beta$ HP1-containing heterochromatic network but strongly support the hypothesis that both proteins possibly display distinct functional roles during endodermal differentiation. Accordingly, we observed a dynamic relocation of Parp-2 to centromeric regions during $\operatorname{PrE}$ differentiation, where it colocalizes with TIF1 $\beta$. In addition, Parp-2-TIF1 $\beta$ association is maintained in $\operatorname{PrE}$ cells. In contrast, Parp-1 subcellular localization remains unaffected throughout differentiation.

We next evaluated the importance of poly(ADPribosyl)ation in the complex. Despite the high $80 \%$ sequence identity and structural similarities among all three HP1 isotypes, we identified a selective poly(ADPribosyl)ation of HP1 $\alpha$ by both Parp-2 and Parp-1. This result suggests a specific direct role of Parp activity in the modulation of higher-order chromatin structures and molecular interactions involving $\mathrm{HP} 1 \alpha$. The mechanism by which Parp-2 and Parp-1 control HP1 $\alpha$ function involves both a covalent heteromodification and a noncovalent binding of poly(ADP-ribose) to HP1 $1 \alpha$. By targeting the hinge domain of $\mathrm{HP} 1 \alpha$, both ways of regulation can help to adjust various hinge-specific functions of HP1 $\alpha$. It is well recognized that the RNA-binding activity of HP1 $\alpha$ that resides within its central hinge domain contributes to its recognition of pericentric HC $(36,37)$. Thus, the effect of HP1 $\alpha$ poly(ADP-ribosyl)ation would be basically to modulate its ability to bind HC, owing to electrostatic repulsion of the negatively charged ADP-ribose polymers present on $\mathrm{HP} 1 \alpha$ from RNA. The normal accumulation of HP1 $\alpha$ on pericentric HC observed in shParp-2 and shParp-1 cells could then be assigned to the compensating meH3K9-binding property of the CD of HP1 $\alpha$. Alternatively, the modification of the hinge domain could modulate the contact of HP1 $\alpha$ with selective partners inside the Parp-1-Parp-2-TIF1 $\beta-H P 1$ complex that would reflect a dynamic equilibrium and important regulatory events occurring in this heterochromatic protein network. Accordingly, we found reduced coimmunoprecipitation of both Parp-2 and HP1 $\alpha$ with TIF1 $\beta$ in the presence of the Parp inhibitor. Altogether it is tempting to propose Parp-1, Parp-2, and poly(ADP-ribose) as auxiliary factors that contribute to the dynamic nature of HP1 either 1) by facilitating its association/ dissociation activity to chromatin, in addition to the previously described histone methyl transferases ACF1 or SUVAR39 (38) or 2) by modulating the protein interaction network at pericentric HC. As such, both Parps could regulate various HP1-dependent processes, including 1) transcriptional silencing of HC (39, $40)$; 2) its participation in kinetochore formation and maintenance during chromosome segregation (41); or 3) its role in HC dynamics during DNA replication (5).

\section{Distinct functions of Parp-2 and Parp-1 in endodermal differentiation and TIF1 $\beta$ dependent transcriptional activity}

Despite the established shared functions of Parp-1 and Parp-2 in cellular response to DNA damage and a similar contribution in the maintenance of $\mathrm{HC}$ integrity, both enzymes have distinct DNA and/or protein targets, which suggests that they might also play unique functions that have only started to be clarified. In the present study, we provide direct evidence that Parp-2 and Parp-1 display key specific functions throughout endodermal differentiation even though both proteins poly(ADP-ribosyl)ate HP1 $\alpha$.

The dynamic accumulation of Parp-2 to centromeric regions in PrE cells, where it colocalizes and interacts with TIF1 $\beta$, combined with the observation that the depletion of Parp-2 or the inhibition of its activity significantly impairs the relocation of TIF $1 \beta$ to nuclear foci, provide compelling evidence that Parp-2 and the associated poly(ADP-ribose) synthesis participate in the targeting of TIF1 $\beta$ to pericentric HC. One major mechanism by which TIF1 $\beta$ is targeted to centromeric regions is through HP1 interaction (30). This association is required for terminal differentiation of $\mathrm{F} 9$ to $\mathrm{PE}$ and VE cells (19). The finding that TIF1 $\beta-H P 1$ association is not significantly disrupted in Parp-2-depleted cells argues for the existence of an additional Parp-2regulated process involved in TIF1 $\beta$ compartmentalization during cellular differentiation. Accordingly, we show here that compromised TIF1 $\beta$ heterochromatic targeting is associated with impaired differentiation of shParp-2 into PrE cells, whereas terminal differentiation of the remaining cells is not affected. These 


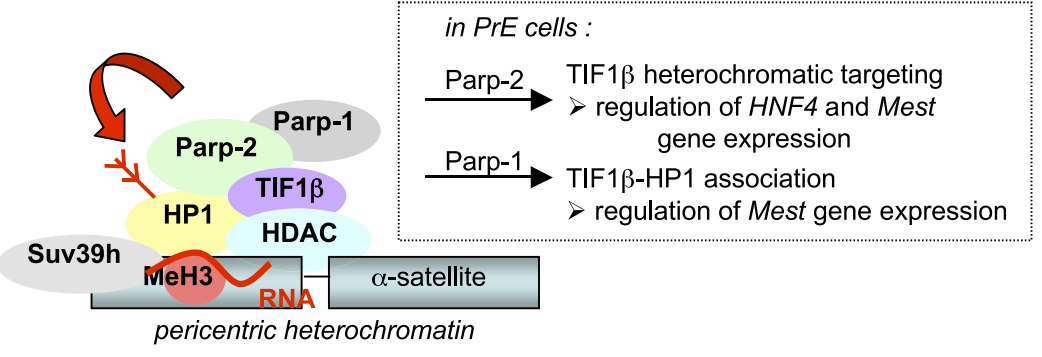

Figure 8. Model describing Parp-1 and Parp-2 as new components of the heterochromatic protein network at pericentric HC in PrE cells. By poly(ADP-ribosyl)ating HP1 $\alpha$, Parp-2 and Parp-1 can be defined as new actors of the HP1-mediated histone subcode required for TIF1 $\beta$ relocation on heterochromatic foci and TIF1 $\beta-H P 1 \alpha$ association throughout endodermal differentiation, respectively. Both ways contribute to the expression of the endoderm-specific gene HNF4 and the mesoderm-specific gene Mest. In addition, the formation of this complex in undifferentiated F9 stem cells suggests additional important roles played by ADP-ribose modifications in controlling chromatin structure and activity during cell proliferation and/or division. Some of these effects might be mediated by HP1 $\alpha$ and/or TIF1 $\beta$.

observations indicate key functions of Parp-2 in initiating differentiation. It is conceivable that through TIF1 $\beta$ HC selective targeting, Parp-2 controls the expression of TIF1 $\beta$-dependent yet-to-be-identified genes involved in early endodermal differentiation. In this respect, it is noteworthy that Parp-2 has recently been shown to associate with and to act as a transcriptional cofactor for PPAR $\gamma$ during adipocyte differentiation (27) and for TTF-1 during lung development (42). In addition, we found a severe down-regulation of the endodermspecific gene HNF4 in Parp-2-depleted and Parp-1-Parp2-depleted cells similar to that previously observed in TIF1 $\beta^{\mathrm{HP} 1 \mathrm{box} /-}$ cells but not in Parp-1-depleted cells. Therefore, Parp-2 participates in the regulation of TIF1 $\beta$ functions, including HC targeting and expression of endoderm-specific genes through a process that might be independent of TIF1 $\beta$-HP1 interaction.

In contrast to Parp-2, we show that Parp-1 and the associated polymerizing activity are crucial for terminal differentiation of F9 cells into PE or VE cells even though Parp-1 does not interact with $\mathrm{HP} 1 \alpha$ and only weakly binds to TIF1 $\beta$. Furthermore, Parp- 1 is at least partly required for stable HP1-TIF $1 \beta$ interaction. Given the essential role of TIF1 $\beta$-HP1 interaction in terminal differentiation (19), it seems likely that the abrogated differentiation of shParp-1 F9 cells to PE or VE cells could be a consequence of the unstable TIF1 $\beta$-HP1 association observed in these cells. However, alternative roles of Parp-1 dependent-poly(ADP-ribosyl)ation of HP1 $\alpha$ that might govern differentiation appear to be involved as a significant level of TIF1 $\beta-\mathrm{HP} 1$ interaction remained reproducibly detected in shParp-1 F9 that is sufficient for the wild-type-like expression of HNF4.

Together, this work highlights key distinct functions of Parp-1 and Parp-2 in endodermal differentiation, although redundant activities cannot be excluded, as indicated by the similar up-regulation of the mesoderm-specific Mest gene in the absence of either Parp-1, Parp-2, or both proteins.

\section{Toward a role of Parp-2 and Parp-1 in the histone subcode}

It is well established that the dynamic regulation of chromatin structure and function is accomplished by a tuned combination of histone modifications, defined as the "histone code" (3). In recent studies, poly(ADPribosyl)ation is turning out to be another of the many global epigenetic modulators involved in chromatin dynamics during physiological processes (28).

More recently, a histone subcode hypothesis has been reported, which predicts that the posttranslational modification of HP1 provides a second regulatory layer of the chromatin code involved in transcriptional repression (9). HP1 proteins can be extensively modified by phosphorylation, acetylation, methylation, sumoylation, and ubiquitination, similar to histones. Here we identified a selective poly(ADP-ribosyl)ation of HP1 $\alpha$ by both Parp- 1 and Parp-2, thus describing PAR as an emerging new modification of subcode proteins. In addition, this property points out Parp- 1 and Parp-2 as new regulators of the histone subcode. The next challenge will be to identify the site-specific residue poly(ADP-ribosyl)ated in HP1 $\alpha$, with the aim of further deciphering the tight regulatory network that regulates these highly similar proteins.

In conclusion, the work described here identifies another step toward the role of TIF1 $\beta-H P 1$ association in endodermal differentiation, defined by distinct physical interactions of Parp- 1 and Parp-2 with TIF1 $\beta$ and HP1 isotypes and a selective poly(ADP-ribosyl)ation of HP1 $\alpha$. A model describing Parp-1 and Parp-2 as essential players in the histone subcode can be proposed in which the selective poly(ADP-ribosyl)ation of HP1 $\alpha$ is a major regulatory event required for TIF1 $\beta$ relocation on heterochromatic foci and TIF1 $\beta$-HP1 $\alpha$-mediated transcriptional activity throughout differentiation (Fig. 8). Identifying the mechanism by which Parp activity is spontaneously induced during this process, as shown in Supplemental Fig. 3, remains an exciting challenge. In addition, these findings reinforce previous evidence that PAR plays fundamental roles in pericentric $\mathrm{HC}$ structure and integrity and open the way toward forthcoming fascinating issues aimed at understanding its contribution in HP1-mediated centromere function and cell division.

We thank V. Schreiber for critical reading of the manuscript and A. van Dorsselear for access to the proteomic platform. This work was supported by Association pour la Recherche Contre le Cancer, Ligue Nationale Contre le Cancer et Comité Régional, Centre National de la Recherche Scientifique, and Agence Nationale de la Recherche. 


\section{REFERENCES}

1. Schneider, R., and Grosschedl, R. (2007) Dynamics and interplay of nuclear architecture, genome organization, and gene expression. Genes Dev. 21, 3027-3043

2. Narlikar, G. J., Fan, H. Y., and Kingston, R. E. (2002) Cooperation between complexes that regulate chromatin structure and transcription. Cell 108, 475-487

3. Jenuwein, T., and Allis, C. D. (2001) Translating the histone code. Science 293, 1074-1080

4. Grewal, S. I., and Jia, S. (2007) Heterochromatin revisited. Nat. Rev. Genet. 8, 35-46

5. Maison, C., and Almouzni, G. (2004) HP1 and the dynamics of heterochromatin maintenance. Nat. Rev. Mol. Cell Biol. 5, 296304

6. Eissenberg, J. C., and Elgin, S. C. (2000) The HP1 protein family: getting a grip on chromatin. Curr. Opin. Genet. Dev. 10, 204-210

7. Lomberk, G., Wallrath, L., and Urrutia, R. (2006) The heterochromatin protein 1 family. Genome Biol. 7, 228

8. Singh, P. B., and Georgatos, S. D. (2002) HP1: facts, open questions, and speculation. J. Struct. Biol. 140, 10-16

9. Lomberk, G., Bensi, D., Fernandez-Zapico, M. E., and Urrutia, R. (2006) Evidence for the existence of an HP1-mediated subcode within the histone code. Nat. Cell Biol. 8, 407-415

10. Le Douarin, B., Nielsen, A. L., Garnier, J. M., Ichinose, H., Jeanmougin, F., Losson, R., and Chambon, P. (1996) A possible involvement of TIF1 alpha and TIF1 beta in the epigenetic control of transcription by nuclear receptors. EMBO J. 15, $6701-6715$

11. Nielsen, A. L., Ortiz, J. A., You, J., Oulad-Abdelghani, M., Khechumian, R., Gansmuller, A., Chambon, P., and Losson, R. (1999) Interaction with members of the heterochromatin protein 1 (HP1) family and histone deacetylation are differentially involved in transcriptional silencing by members of the TIF1 family. EMBO J. 18, 6385-6395

12. Ryan, R. F., Schultz, D. C., Ayyanathan, K., Singh, P. B. Friedman, J. R., Fredericks, W. J., and Rauscher, F. J., 3rd. (1999) KAP-1 corepressor protein interacts and colocalizes with heterochromatic and euchromatic HP1 proteins: a potential role for Kruppel-associated box-zinc finger proteins in heterochromatin-mediated gene silencing. Mol. Cell Biol. 19, 43664378

13. Sripathy, S. P., Stevens, J., and Schultz, D. C. (2006) The KAP1 corepressor functions to coordinate the assembly of de novo HP1-demarcated microenvironments of heterochromatin required for KRAB zinc finger protein-mediated transcriptional repression. Mol. Cell Biol. 26, 8623-8638

14. Schultz, D. C., Friedman, J. R., and Rauscher, F. J., 3rd. (2001) Targeting histone deacetylase complexes via KRAB-zinc finger proteins: the PHD and bromodomains of KAP-1 form a cooperative unit that recruits a novel isoform of the Mi-2alpha subunit of NuRD. Genes Dev. 15, 428-443

15. Ayyanathan, K., Lechner, M. S., Bell, P., Maul, G. G., Schultz, D. C., Yamada, Y., Tanaka, K., Torigoe, K., and Rauscher, F. J., 3rd. (2003) Regulated recruitment of HP1 to a euchromatic gene induces mitotically heritable, epigenetic gene silencing: a mammalian cell culture model of gene variegation. Genes Dev. 17, 1855-1869

16. Cammas, F., Mark, M., Dolle, P., Dierich, A., Chambon, P., and Losson, R. (2000) Mice lacking the transcriptional corepressor TIFlbeta are defective in early postimplantation development. Development 127, 2955-2963

17. Weber, P., Cammas, F., Gerard, C., Metzger, D., Chambon, P., Losson, R., and Mark, M. (2002) Germ cell expression of the transcriptional co-repressor TIF1beta is required for the maintenance of spermatogenesis in the mouse. Development 129, $2329-2337$

18. Strickland, S., and Mahdavi, V. (1978) The induction of differentiation in teratocarcinoma stem cells by retinoic acid. Cell 15, 393-403

19. Cammas, F., Herzog, M., Lerouge, T., Chambon, P., and Losson, R. (2004) Association of the transcriptional corepressor TIF1beta with heterochromatin protein 1 (HP1): an essential role for progression through differentiation. Genes Dev. 18, 2147-2160
20. Schreiber, V., Dantzer, F., Ame, J. C., and de Murcia, G. (2006) Poly(ADP-ribose): novel functions for an old molecule. Nat. Rev. Mol. Cell Biol. 7, 517-528

21. Menissier de Murcia, J., Ricoul, M., Tartier, L., Niedergang, C., Huber, A., Dantzer, F., Schreiber, V., Ame, J. C., Dierich, A., LeMeur, M., Sabatier, L., Chambon, P., and de Murcia, G. (2003) Functional interaction between PARP-1 and PARP-2 in chromosome stability and embryonic development in mouse. EMBO J. 22, 2255-2263

22. Dantzer, F., Giraud-Panis, M. J., Jaco, I., Ame, J. C., Schultz, I., Blasco, M., Koering, C. E., Gilson, E., Menissier-de Murcia, J., de Murcia, G., and Schreiber, V. (2004) Functional interaction between poly(ADP-Ribose) polymerase 2 (PARP-2) and TRF2: PARP activity negatively regulates TRF2. Mol. Cell Biol. 24, $1595-1607$

23. Saxena, A., Saffery, R., Wong, L. H., Kalitsis, P., and Choo, K. H. (2002) Centromere proteins Cenpa, Cenpb, and Bub3 interact with poly(ADP-ribose) polymerase-1 protein and are poly(ADPribosyl)ated. J. Biol. Chem. 277, 26921-26926

24. Saxena, A., Wong, L. H., Kalitsis, P., Earle, E., Shaffer, L. G., and Choo, K. H. (2002) Poly(ADP-ribose) polymerase 2 localizes to mammalian active centromeres and interacts with PARP-1, Cenpa, Cenpb and Bub3, but not Cenpc. Hum. Mol. Genet. 11, $2319-2329$

25. Meder, V. S., Boeglin, M., de Murcia, G., and Schreiber, V (2005) PARP-1 and PARP-2 interact with nucleophosmin/B23 and accumulate in transcriptionally active nucleoli. J. Cell Sci. 118, 211-222

26. Ju, B. G., Solum, D., Song, E. J., Lee, K. J., Rose, D. W., Glass, C. K., and Rosenfeld, M. G. (2004) Activating the PARP-1 sensor component of the groucho/ TLE1 corepressor complex mediates a CaMKinase IIdelta-dependent neurogenic gene activation pathway. Cell 119, 815-829

27. Bai, P., Houten, S. M., Huber, A., Schreiber, V., Watanabe, M., Kiss, B., de Murcia, G., Auwerx, J., and Menissier-de Murcia, J. (2007) PARP-2 controls adipocyte differentiation and adipose tissue function through the regulation of the activity of the RXR/PPARgamma heterodimer. J. Biol. Chem. 282, $37738-$ 37746

Dantzer, F., Mark, M., Quenet, D., Scherthan, H., Huber, A., Liebe, B., Monaco, L., Chicheportiche, A., Sassone-Corsi, P., de Murcia, G., and Menissier-de Murcia, J. (2006) Poly(ADPribose) polymerase-2 contributes to the fidelity of male meiosis I and spermiogenesis. Proc. Natl. Acad. Sci. U. S. A. 103, $14854-$ 14859

29. Yelamos, J., Monreal, Y., Saenz, L., Aguado, E., Schreiber, V., Mota, R., Fuente, T., Minguela, A., Parrilla, P., de Murcia, G., Almarza, E., Aparicio, P., and Menissier-de Murcia, J. (2006) PARP-2 deficiency affects the survival of $\mathrm{CD} 4+\mathrm{CD} 8+$ doublepositive thymocytes. EMBO J. 25, 4350-4360

30. Cammas, F., Oulad-Abdelghani, M., Vonesch, J. L., Huss-Garcia, Y., Chambon, P., and Losson, R. (2002) Cell differentiation induces TIF1beta association with centromeric heterochromatin via an HP1 interaction. J. Cell Sci. 115, 3439-3448

31. Miguet, L., Pacaud, K., Felden, C., Hugel, B., Martinez, M. C., Freyssinet, J. M., Herbrecht, R., Potier, N., van Dorsselaer, A., and Mauvieux, L. (2006) Proteomic analysis of malignant lymphocyte membrane microparticles using double ionization coverage optimization. Proteomics 6, 153-171

32. Farmer, H., McCabe, N., Lord, C. J., Tutt, A. N., Johnson, D. A., Richardson, T. B., Santarosa, M., Dillon, K. J., Hickson, I., Knights, C., Martin, N. M., Jackson, S. P., Smith, G. C., and Ashworth, A. (2005) Targeting the DNA repair defect in BRCA mutant cells as a therapeutic strategy. Nature 434, 917-921

33. Schreiber, V., Ame, J. C., Dolle, P., Schultz, I., Rinaldi, B., Fraulob, V., Menissier-de Murcia, J., and de Murcia, G. (2002) Poly(ADP-ribose) polymerase-2 (PARP-2) is required for efficient base excision DNA repair in association with PARP-1 and XRCC1. J. Biol. Chem. 277, 23028-23036

34. Nusinow, D. A., Hernandez-Munoz, I., Fazzio, T. G., Shah, G. M., Kraus, W. L., and Panning, B. (2007) Poly(ADP-ribose) polymerase 1 is inhibited by a histone H2A variant, MacroH2A, and contributes to silencing of the inactive $\mathrm{X}$ chromosome. J. Biol. Chem. 282, 12851-12859

35. Aldinucci, A., Gerlini, G., Fossati, S., Cipriani, G., Ballerini, C. Biagioli, T., Pimpinelli, N., Borgognoni, L., Massacesi, L., Moroni, F., and Chiarugi, A. (2007) A key role for poly(ADP-ribose) 
polymerase-1 activity during human dendritic cell maturation. J. Immunol. 179, 305-312

36. Maison, C., Bailly, D., Peters, A. H., Quivy, J. P., Roche, D. Taddei, A., Lachner, M., Jenuwein, T., and Almouzni, G. (2002) Higher-order structure in pericentric heterochromatin involves a distinct pattern of histone modification and an RNA component. Nat. Genet. 30, 329-334

37. Muchardt, C., Guilleme, M., Seeler, J. S., Trouche, D., Dejean, A., and Yaniv, M. (2002) Coordinated methyl and RNA binding is required for heterochromatin localization of mammalian HPlalpha. EMBO Rep. 3, 975-981

38. Eskeland, R., Eberharter, A., and Imhof, A. (2007) HP1 binding to chromatin methylated at H3K9 is enhanced by auxiliary factors. Mol. Cell Biol. 27, 453-465

39. Nielsen, S. J., Schneider, R., Bauer, U. M., Bannister, A. J., Morrison, A., O'Carroll, D., Firestein, R., Cleary, M., Jenuwein,
T., Herrera, R. E., and Kouzarides, T. (2001) Rb targets histone H3 methylation and HP1 to promoters. Nature 412, 561-565

40. Richards, E. J., and Elgin, S. C. (2002) Epigenetic codes for heterochromatin formation and silencing: rounding up the usual suspects. Cell 108, 489-500

41. Obuse, C., Iwasaki, O., Kiyomitsu, T., Goshima, G., Toyoda, Y., and Yanagida, M. (2004) A conserved Mis12 centromere complex is linked to heterochromatic HP1 and outer kinetochore protein Zwint-1. Nat. Cell Biol. 6, 1135-1141

42. Maeda, Y., Hunter, T. C., Loudy, D. E., Dave, V., Schreiber, V., and Whitsett, J. A. (2006) PARP-2 interacts with TTF-1 and regulates expression of surfactant protein-B. J. Biol. Chem. 281 , $9600-9606$

Received for publication May 13, 2008. Accepted for publication June 26, 2008.

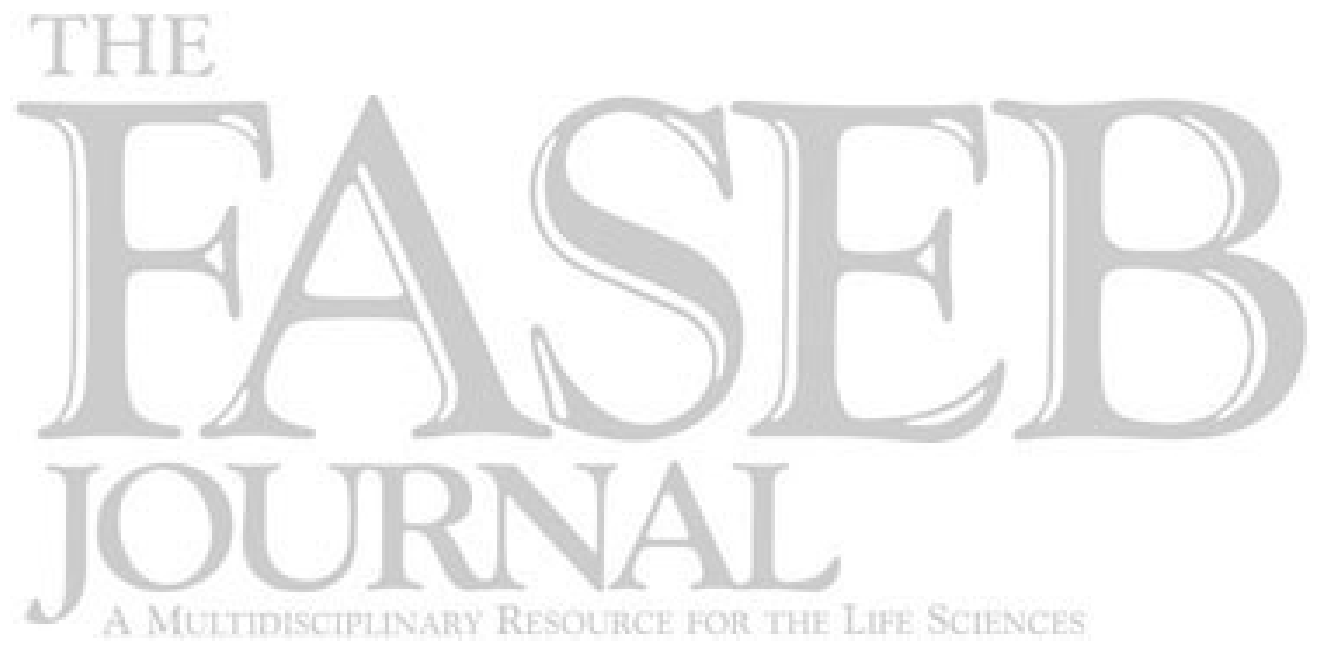




\section{AUTHOR QUERIES}

\section{AUTHOR PLEASE ANSWER ALL QUERIES}

1-Please spell out IDG, ESBS, IGBMC, and ECPM. ????

2-Per journal style, key words must not repeat terms that appear in the title or short running title. Please add up to 5 additional key words. ????

3-Per journal style, first citation of materials, equipment, and software used should include supplier name and location (city and state or country). Please confirm or revise this information where it has been added throughout Materials and Methods. ????

4-Please list supplier name and location for OpenLab software. ????

5-Please ensure that correspondence address is a complete postal mailing address. ???? 